\title{
Concomitant SK current activation and sodium current inhibition cause J wave syndrome
}

\author{
Mu Chen, ${ }^{1,2}$ Dong-Zhu Xu, ${ }^{1,3}$ Adonis Z. Wu, ${ }^{1}$ Shuai Guo, ${ }^{1,4}$ Juyi Wan, ${ }^{1,5}$ Dechun Yin, ${ }^{1,4}$ \\ Shien-Fong Lin, ${ }^{1,6}$ Zhenhui Chen, ${ }^{1}$ Michael Rubart-von der Lohe, ${ }^{1,7}$ Thomas H. Everett IV, ${ }^{1}$ Zhilin Qu, ${ }^{8}$ \\ James N. Weiss, ${ }^{8}$ and Peng-Sheng Chen \\ ${ }^{1}$ Krannert Institute of Cardiology and Division of Cardiology, Department of Medicine, Indiana University School of \\ Medicine, Indianapolis, Indiana, USA. ²Department of Cardiology, Xinhua Hospital, School of Medicine, Shanghai Jiao Tong \\ University, Shanghai, China. ${ }^{3}$ Cardiovascular Division, Institute of Clinical Medicine, Faculty of Medicine, University of \\ Tsukuba, Tsukuba, Japan. ${ }^{4}$ Department of Cardiology, First Affiliated Hospital of Harbin Medical University, Harbin, China. \\ ${ }^{5}$ Department of Cardiothoracic Surgery, Affiliated Hospital of Southwest Medical University, Luzhou, China. ${ }^{6}$ Institute of \\ Biomedical Engineering, College of Electrical and Computer Engineering, National Chiao Tung University, Hsin-Chu, Taiwan. \\ ${ }^{7}$ Department of Pediatrics, Indiana University School of Medicine, Indianapolis, Indiana, USA. ${ }^{8}$ Departments of Medicine \\ (Cardiology) and Physiology, University of California, Los Angeles, California, USA.
}

The mechanisms of J wave syndrome (JWS) are incompletely understood. Here, we showed that the concomitant activation of small-conductance calcium-activated potassium (SK) current $\left(I_{\mathrm{KAS}}\right)$ and inhibition of sodium current by cyclohexyl-[2-(3,5-dimethyl-pyrazol-1-yl)-6-methyl-pyrimidin4-yl]-amine (CyPPA) recapitulate the phenotypes of JWS in Langendorff-perfused rabbit hearts. CyPPA induced significant I wave elevation and frequent spontaneous ventricular fibrillation (SVF), as well as sinus bradycardia, atrioventricular block, and intraventricular conduction delay. $I_{\text {KAS }}$ activation by CyPPA resulted in heterogeneous shortening of action potential (AP) duration (APD) and repolarization alternans. CyPPA inhibited cardiac sodium current $\left(I_{\mathrm{Na}}\right)$ and decelerated AP upstroke and intracellular calcium transient. SVFs were typically triggered by short-coupled premature ventricular contractions, initiated with phase 2 reentry and originated more frequently from the right than the left ventricles. Subsequent $I_{\text {KAS }}$ blockade by apamin reduced J wave elevation and eliminated SVF. $\beta$-Adrenergic stimulation was antiarrhythmic in CyPPA-induced electrical storm. Like CyPPA, hypothermia $\left(32.0^{\circ} \mathrm{C}\right)$ also induced J wave elevation and SVF. It facilitated negative calcium-voltage coupling and phase 2 repolarization alternans with spatial and electromechanical discordance, which were ameliorated by apamin. These findings suggest that $I_{\text {KAS }}$ activation contributes to the development of JWS in rabbit ventricles.

Conflict of interest: The authors have declared that no conflict of interest exists.

Submitted: May 21, 2018

Accepted: July 17, 2018

Published: August 23, 2018

Reference information: JCI Insight. 2018;3(16):e122329. https://doi.org/10.1172/jici. insight.122329.

\section{Introduction}

The J wave syndromes (JWS), consisting of the Brugada syndrome (BrS) and early repolarization (ER) syndrome (ERS), are characterized by accentuated J waves on the electrocardiogram (ECG) and the vulnerability to life-threatening ventricular arrhythmias in young adults with no apparent structural heart diseases (1). The development of JWS is attributed to the loss of function of the depolarizing currents (such as sodium current $I_{\mathrm{Na}}$ and L-type calcium current $I_{\mathrm{C}, \mathrm{L}}$ ) with the concomitant gain of function of the repolarizing currents (such as transient outward potassium current $I_{\mathrm{to}}$ and adenosine triphosphate sensitive potassium current $\left.I_{\text {K-ATP }}\right)(1)$. The imbalanced ion channel dysfunction leads to heterogeneous early repolarization as well as depolarization abnormalities and slow conduction, thus facilitating phase 2 reentry (P2R) and ventricular fibrillation (VF) (1). JWS are considered to be genetic ion channelopathies due to the variants found in genes encoding these cardiac ion channels as well as other regulatory proteins (1), and also occur in acquired conditions, such as hypothermia. A common finding of both the congenital and acquired JWS is reduced $I_{\mathrm{Na}}$. Blockers of $I_{\mathrm{Na}}$ such as ajmaline and flecainide are commonly used to unmask the BrS (2). Hypothermia, which causes J wave elevation (Osborn wave), is also known to be associated with significantly reduced peak $I_{\mathrm{Na}}(3,4)$. However, genetically engineered pigs that expresses SCN5A E558X variant 
identified from a patient with BrS exhibited only conduction defects, but no J wave elevation at baseline or after flecainide administration (5). These data suggest that reduction of $I_{\mathrm{Na}}$ alone, in the absence of a significant transient outward repolarizing $\mathrm{K}^{+}$current, does not cause JWS.

Apamin-sensitive small-conductance calcium-activated potassium (SK) current $\left(I_{\mathrm{KAS}}\right)$ is important in cardiac repolarization and arrhythmogenesis in multiple physiological and pathological conditions (6-8). Moreover, because its conductance tracks the $\mathrm{Ca}^{2+}$ transient, it behaves functionally like a transient outward $\mathrm{K}^{+}$current. In recent human studies, genetic polymorphisms of $K C N N 3$ (encodes SK3) and $K C N N 2$ (encodes SK2) were found to be associated with atrial fibrillation, ventricular tachyarrhythmias, and sudden cardiac death $(9,10)$. Overexpression of $K C N N 3$ in mice caused bradycardia, atrioventricular (AV) block, and sudden cardiac death (11). Because a correlation between $I_{\text {KAS }}$ activation and ventricular arrhythmias was discovered just recently in 2011 (6), the relationship between $I_{\text {KAS }}$ and JWS has not yet been investigated. Since $I_{\mathrm{KAS}}$ is most abundantly activated during the early repolarization phase of the action potential (AP) when $I_{\mathrm{Ca}, \mathrm{L}}$ activity, sarcoplasmic calcium release, and intracellular calcium $\left(\mathrm{Ca}_{\mathrm{i}}\right)$ are high, it is reasonable to hypothesize that $I_{\text {KAS }}$ activation could mimic $I_{\text {to }}$ in precipitating arrhythmias seen in JWS.

Cyclohexyl-[2-(3,5-dimethyl-pyrazol-1-yl)-6-methyl-pyrimidin-4-yl]-amine (CyPPA) is the first subtypeselective SK channel positive modifier (12). It also exhibits inhibitory effects on $I_{\mathrm{Na}}$ mediated by $\mathrm{Na}_{\mathrm{v}} 1.2$ (12), but its effects on cardiac $I_{\mathrm{Na}}$ remain unclear. Here, we verified that CyPPA inhibits cardiac $I_{\mathrm{Na}}$ so that the effects of CyPPA are combined with $I_{\mathrm{KAS}}$ activation and $I_{\mathrm{Na}}$ blockade. In this study, we recapitulated JWS phenotypes in CyPPA or hypothermia-treated rabbit whole hearts, which manifested with $\mathrm{J}$ wave elevation and spontaneous ventricular fibrillation (SVF) or spontaneous ventricular tachycardia (SVT), as well as conduction abnormalities. We further showed that apamin, a specific $I_{\text {KAS }}$ blocker (13), eliminated J wave elevation and ventricular arrhythmias.

\section{Results}

CyPPA induces JWS. As shown in Figure 1A, neither J wave nor SVF/SVT was observed at baseline. After $I_{\mathrm{KAS}}$ activation by CyPPA, J point was elevated in all 12 hearts, including 6 type 1 Brugada waves, 2 type 2 Brugada waves, and 4 ER. SVF or SVT developed in 11 of 12 hearts with a total of 128 episodes, which were typically triggered by short-coupled premature ventricular contractions (PVCs, black arrow) (Figure 1B). Atrial tachycardia and different degrees of AV block were also commonly observed. Subsequent $I_{\text {KAs }}$ blockade by apamin attenuated J waves in all hearts. Only 2 episodes of SVF/SVT occurred after apamin. These findings show that $I_{\text {KAS }}$ activation is required for the development of JWS. Figure 1C shows CyPPA induced spontaneous variations of Brugada waves, which were frequently observed in BrS patients. Summary data in Figure 1D shows that the $\mathrm{J}$ point amplitude and the number of SVF/SVT episodes were significantly higher after CyPPA $(0.31 \pm 0.04 \mathrm{mV}$ and $10.4 \pm 4.2)$ than at baseline $(0.07 \pm 0.02 \mathrm{mV}$ and 0.0 $\pm 0.00)$, but decreased after apamin $(0.11 \pm 0.06 \mathrm{mV}$ and $0.2 \pm 0.1, P<0.05$ for all). Thus, in normal rabbit hearts, we recapitulated JWS phenotypes by CyPPA. Excessive $I_{\mathrm{KAS}}$ activation is highly proarrhythmic, while $I_{\mathrm{KAS}}$ blockade is antiarrhythmic.

CyPPA heterogeneously shortens action potential (AP) duration (APD). To understand the mechanisms of the CyPPA-induced JWS, optical mapping was performed to identify the effects of CyPPA on APD (protocol I). As shown in Figure 2A, APD was homogeneous at baseline. CyPPA heterogeneously shortened $\mathrm{APD}_{25}$ at all pacing cycle lengths (PCLs) and led to AP triangulation, indicating heterogeneous $I_{\mathrm{KAS}}$ activation at the early phases of repolarization. Subsequent apamin addition prolonged both $\mathrm{APD}_{25}$ and $\mathrm{APD}_{80}$, reduced $\mathrm{APD}$ heterogeneity, and restored the phase 2 plateau of repolarization. The $\triangle \mathrm{APD}$ maps in Figure $2 \mathrm{~B}$ further strengthened the heterogeneous APD shortening after CyPPA and heterogeneous APD prolongation after apamin. Compared with the left ventricles (LVs), large $\triangle \mathrm{APD}$ zones were more commonly observed in the right ventricles (RVs). With small $\triangle \mathrm{APD}$ islands scattered inside, RVs harbored more arrhythmogenic substrate with the formation of steep APD gradients. Figure $2 \mathrm{C}$ shows a pseudo-electrocardiogram (pECG) and its corresponding $\mathrm{V}_{\mathrm{m}}$ traces from different regions. The abbreviated and early repolarized AP at site 1 was correlated with J point elevation of the pECG. Figure $2 \mathrm{D}$ summarizes that $\mathrm{APD}_{25}$, but not $\mathrm{APD}_{80}$, was significantly abbreviated by CyPPA, while both were significantly prolonged by subsequent apamin at all PCLs.

CyPPA increases AP dynamic instabilities. $\mathrm{T}$ wave alternans is associated with $\mathrm{VF}$ occurrences in $\mathrm{BrS}$ patients (14). To test the effects of CyPPA on repolarization variability, 5 continuous sinus beats were optically mapped. As shown in Figure 3A, APD exhibited spatiotemporal homogeneity at baseline. After CyPPA, AP exhibited increased dispersion with steep APD gradients forming mainly in the RV (red arrow), 


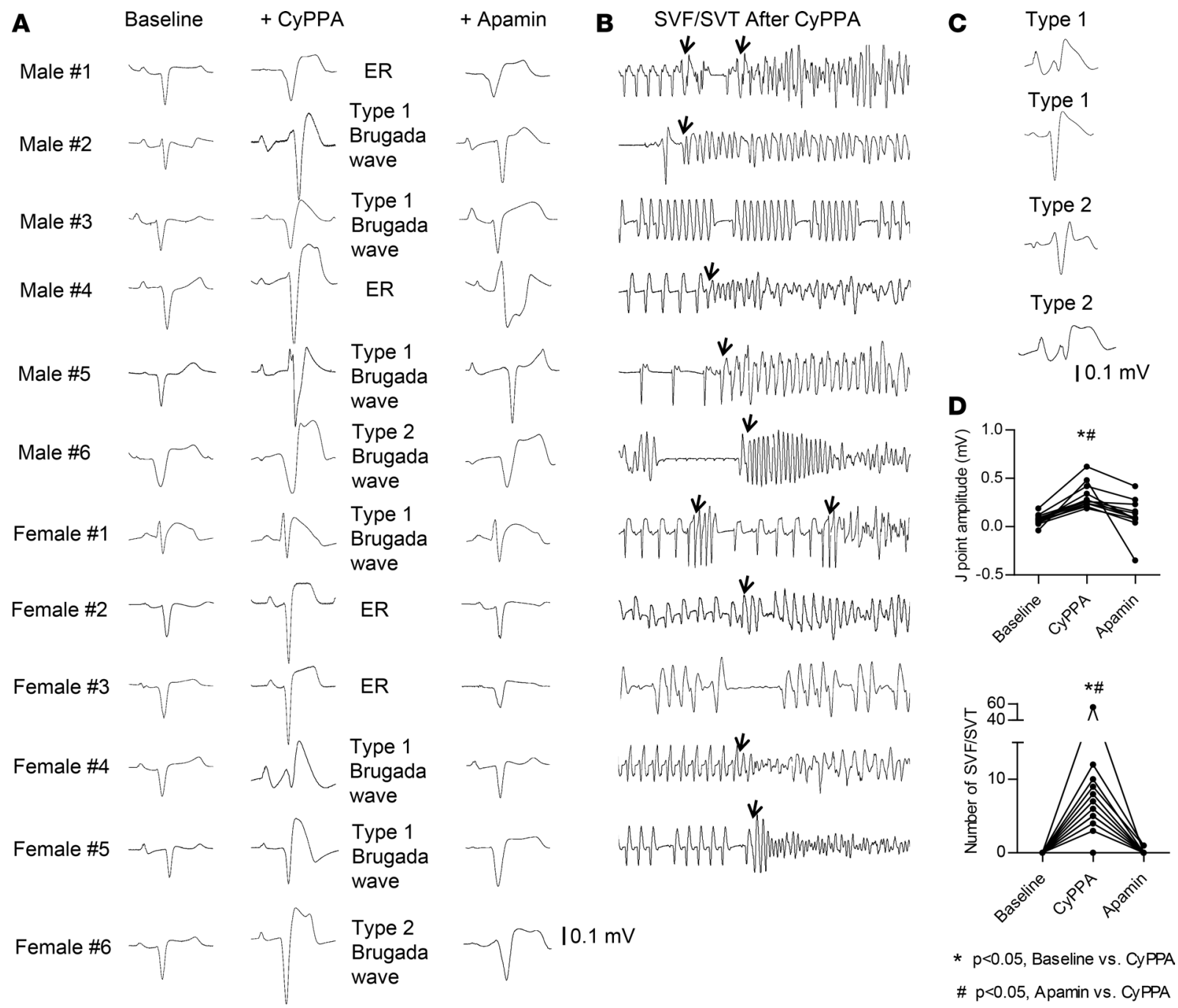

Figure 1. J wave syndrome is induced by CyPPA and suppressed by apamin. (A) pECG during sinus rhythm (protocol I). No J wave was observed at baseline. CyPPA accentuated J waves in all 12 hearts with either Brugada or early repolarization (ER) morphologies. Subsequent apamin attenuated J waves in all hearts. (B) CyPPA induced spontaneous ventricular fibrillation (SVF) or spontaneous ventricular tachycardia (SVT) in 11 of 12 hearts. SVFs were typically triggered by spontaneous short-coupled premature ventricular contractions (PVCs, black arrows). Atrial tachycardia and atrioventricular block were also observed. (C) Spontaneous variations of Brugada waves. (D) Summary of the J point amplitude and the episode numbers of SVF/SVT at baseline, after CyPPA and after apamin. Statistical significance was determined by 1-way ANOVA with Tukey's post hoc test.

which changed dynamically from beat to beat. The spatial heterogeneities and temporal instabilities of AP were attenuated by subsequent exposure to apamin. As further shown in Figure 3B, $\triangle \mathrm{APD}$ between 2 successive beats was minimal at baseline. After CyPPA, $\triangle \mathrm{APD}$ increased prominently with beat-to-beat variations. The large $\triangle \mathrm{APD}$ zones (blue or red) were more frequently located in the RV than the LV. Apamin reduced the AP discordance and instabilities. These results indicate that $I_{\mathrm{KAS}}$ activation is spatiotemporally heterogeneous. The steep APD gradient, increased AP dispersions, and dynamic variations suggest heightened arrhythmogenicity with $I_{\mathrm{KAS}}$ activation, especially in the RV.

Effects of CyPPA on depolarization, conduction, and calcium transient. In addition to repolarization abnormalities, JWS is also associated with abnormal depolarization and conduction delay (15). Therefore, we further evaluated the effects of CyPPA on depolarization, intraventricular conduction, and $\mathrm{Ca}_{\mathrm{i}}$ cycling. Compared with baseline, CyPPA significantly decelerated AP upstroke and slowed intraventricular conduction velocity (CV) at all PCLs (Figure 4, A and B). Voltage clamp study indicated that CyPPA at 10 $\mu \mathrm{mol} / 1$ inhibited $I_{\mathrm{Na}}$ in rabbit ventricular myocytes (Figure 4, C and D, Supplemental Figure 1, and Supplemental Table 1; supplemental material available online with this article; https://doi.org/10.1172/jci. insight.122329DS1). As shown in Figure 4, E and F, CyPPA significantly abbreviated $\mathrm{Ca}_{\mathrm{i}}$ transient duration 
A

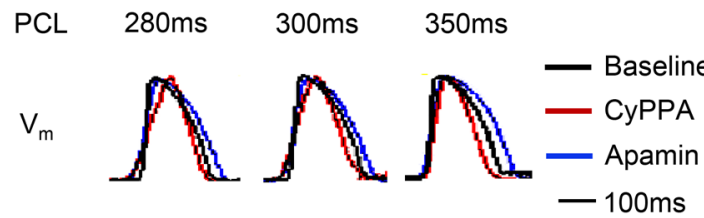

Baseline
$\mathrm{APD}_{25} \mathrm{RV}\left(\begin{array}{l}\kappa_{L A D} \\ \mathrm{LV}\end{array}\right.$

81

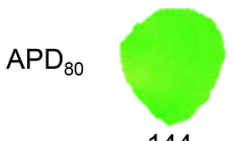

144

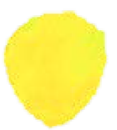

86

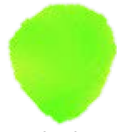

151

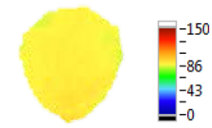

$87 \quad(\mathrm{~ms})$

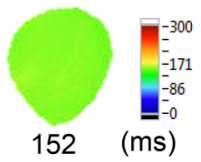

B

$\triangle \mathrm{APD}_{25}$

29

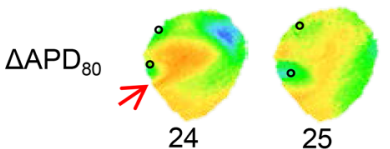

०。
Baseline- CyPPA

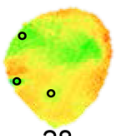

28

25
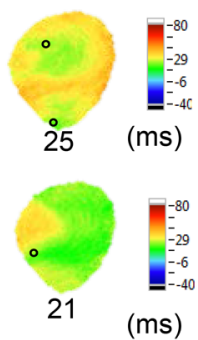

C

CyPPA

$V_{m}$ map
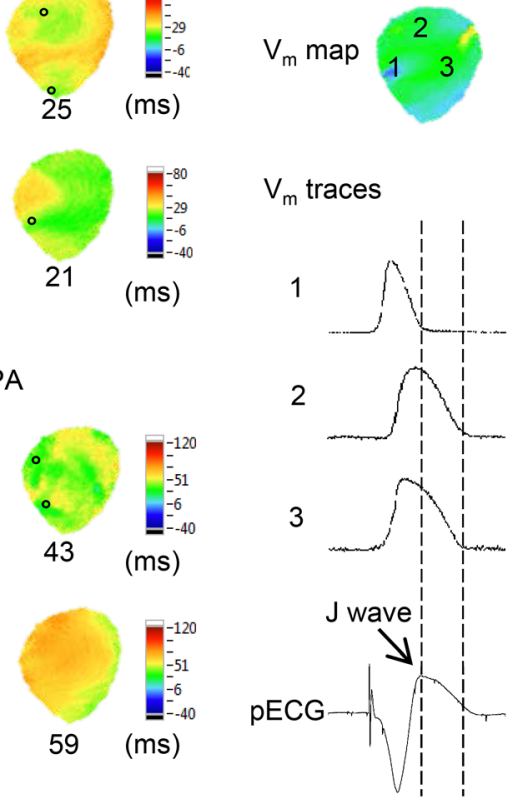

CyPPA
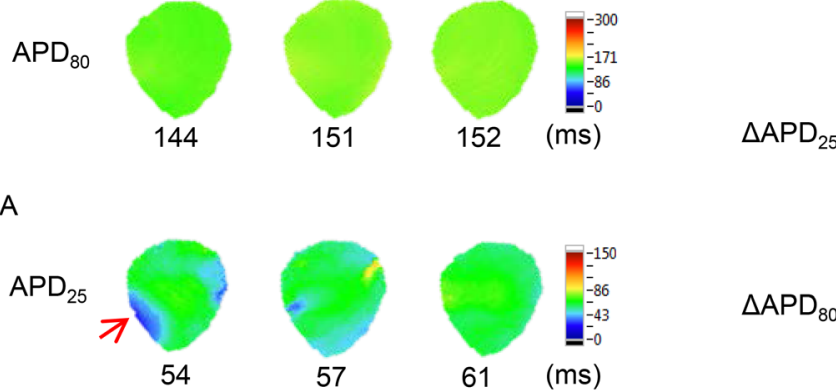

Apamin- CyPPA
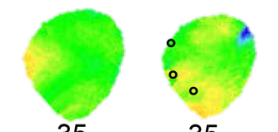

35

43

(ms)

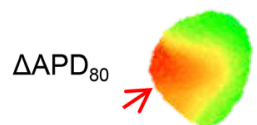

48

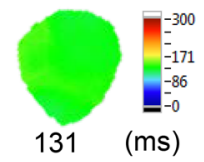

Apamin
$\mathrm{APD}_{25}$

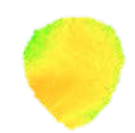

89

$\mathrm{APD}_{80}$

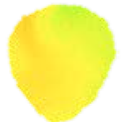

175
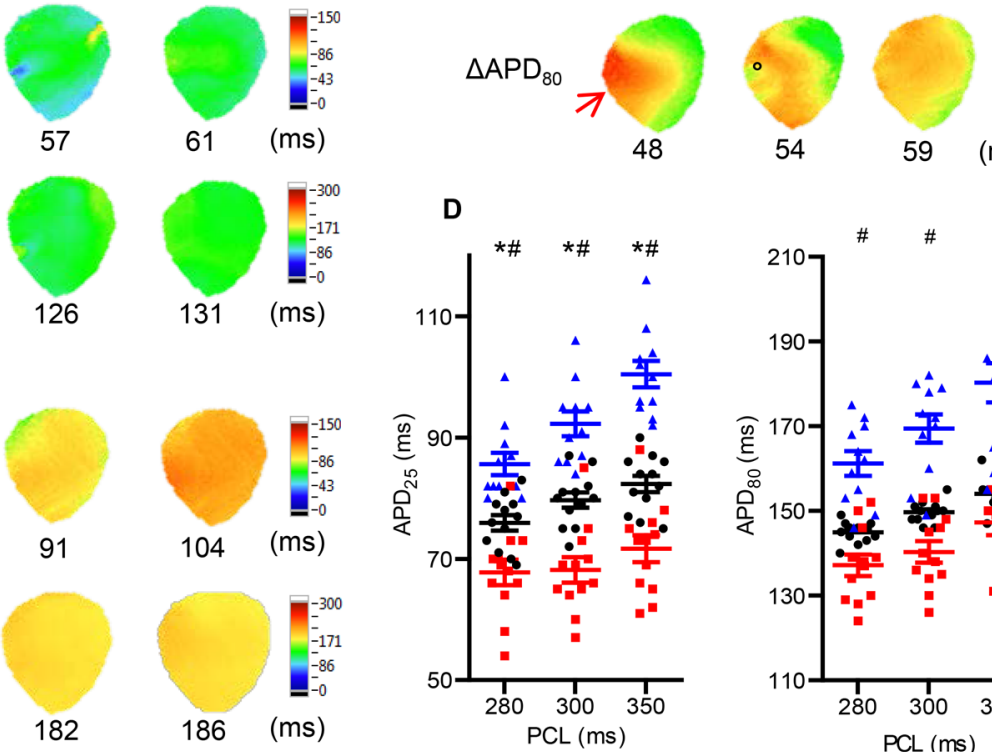

59
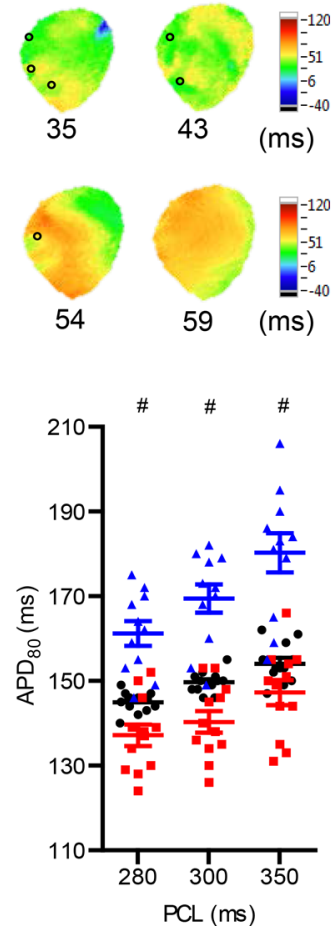

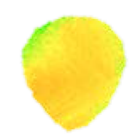

91

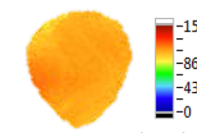

104 (ms)
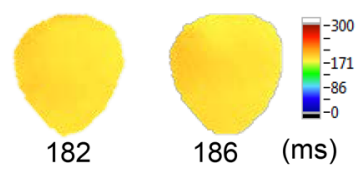

$\mathrm{PCL}(\mathrm{ms})$

PCL (ms)

Figure 2. Effects of CyPPA and apamin on action potential duration (APD). (A) Overlapped $V_{m}$ traces and representative $A P D_{25}$ and $A P D_{80} m a p s$ at baseline, after CyPPA and after apamin with right ventricular (RV) pacing at pacing cycle length (PCL) $280 \mathrm{~ms}, 300 \mathrm{~ms}$, and $350 \mathrm{~ms}$ (protocol I, $n=12$ ). APD exhibited homogeneity at baseline. CyPPA heterogeneously shortened and triangulated APD at all PCLs. The heterogeneity was more severe in the RVs than the left ventricles (LVs). Apamin prolonged APD and restored AP plateau and APD homogeneity. LAD indicates left anterior descending artery. (B) $\triangle A P D_{\text {Baseline-cypPA }}$ and $\triangle A P D_{\text {Apamin-CyPPA }}$ maps display heterogeneous APD shortening after CyPPA and heterogeneous prolongation after apamin. Large $\triangle A P D$ areas (red, orange, or yellow zones) were more likely distributed in the RV with small $\triangle A P D$ spots (green dots marked with ${ }^{\circ}$ ) scattering inside, thus forming a steep APD gradient. (C) pECG and corresponding optical traces recorded at different sites. (D) Summary of $A P D_{25}$ and $A P D_{80}$ at baseline, after $C y P P A$ and after apamin in all 3 PCLs. Data represent mean \pm SEM. Statistical significance was determined by 2-way ANOVA with Bonferroni's post hoc test.

$\left(\mathrm{Ca}_{\mathrm{i}} \mathrm{TD}_{25}\right)$ at all PCLs. Compared with CyPPA, apamin prolonged $\mathrm{Ca}_{\mathrm{i}} \mathrm{TD}_{25}$ at longer PCLs and $\mathrm{Ca}_{\mathrm{i}} \mathrm{TD}_{80}$ at all PCLs. These effects on calcium transient in whole hearts were supported by confocal calcium imaging in fluo-4AM-loaded isolated ventricular cardiomyocytes, showing increased time to peak and decreased decay time after CyPPA, which were prolonged after apamin (Figure 4G).

Arrhythmogenic effects of CyPPA. We further investigated the properties of ventricular arrhythmias induced by CyPPA. Supplemental Figure 2 displays an example of pacing-induced P2R after CyPPA, which was eliminated by subsequent apamin addition. More importantly, we optically captured SVF or SVT that occurred after CyPPA. As shown in Figure 5A, J point was elevated during sinus rhythm before the SVF onset. RR intervals gradually shortened due to type 1 (Wenckebach) second-degree AV block. Two episodes of SVF subsequently initiated from spontaneous short-coupled PVCs, which were confirmed in corresponding membrane potential $\left(\mathrm{V}_{\mathrm{m}}\right)$ traces (Figure $5 \mathrm{~B}$ ). The $\mathrm{V}_{\mathrm{m}}$ map of beat 1 (Figure $5 \mathrm{C}$ ) exhibited an extremely steep APD gradient, providing an enhanced arrhythmogenicity prior to the onset of SVF. The phase map of 
A

Baseline

Beat 1 Beat 2 Beat 3 Beat 4 Beat 5

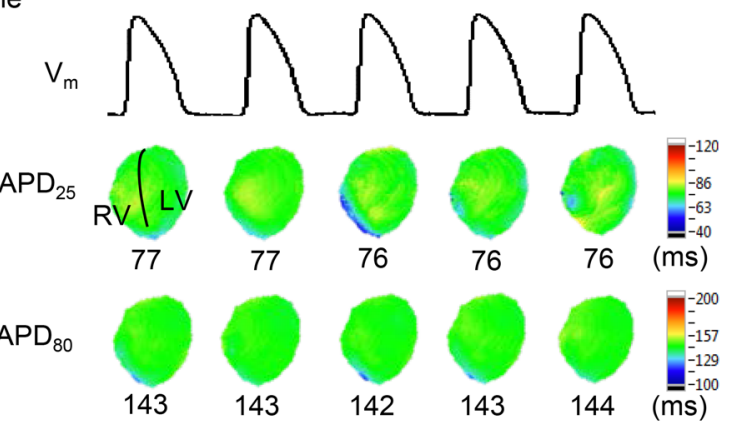

B

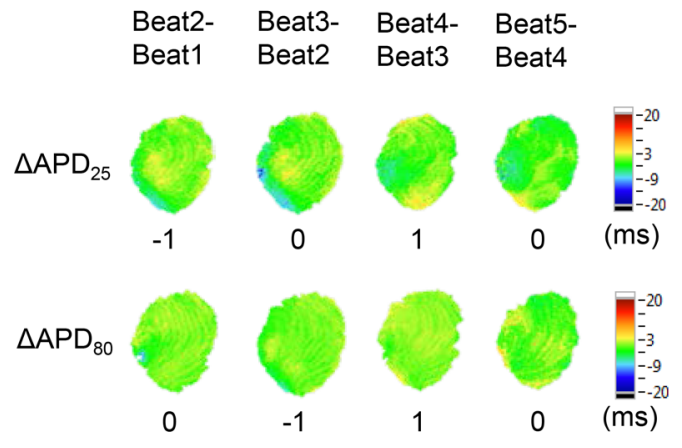

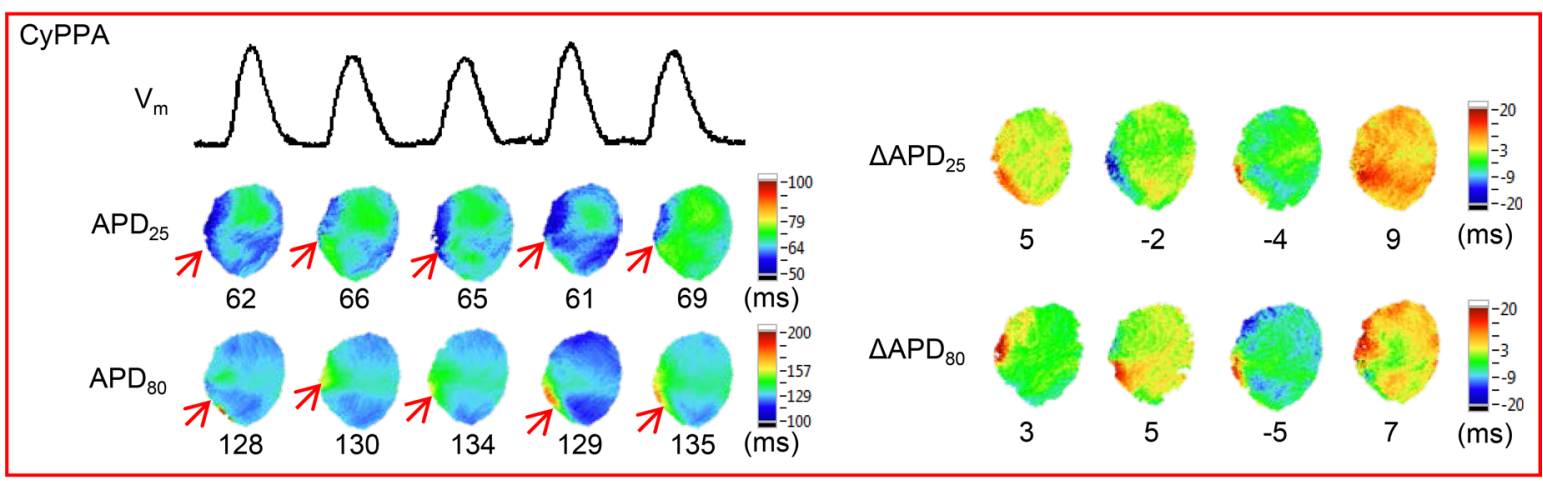
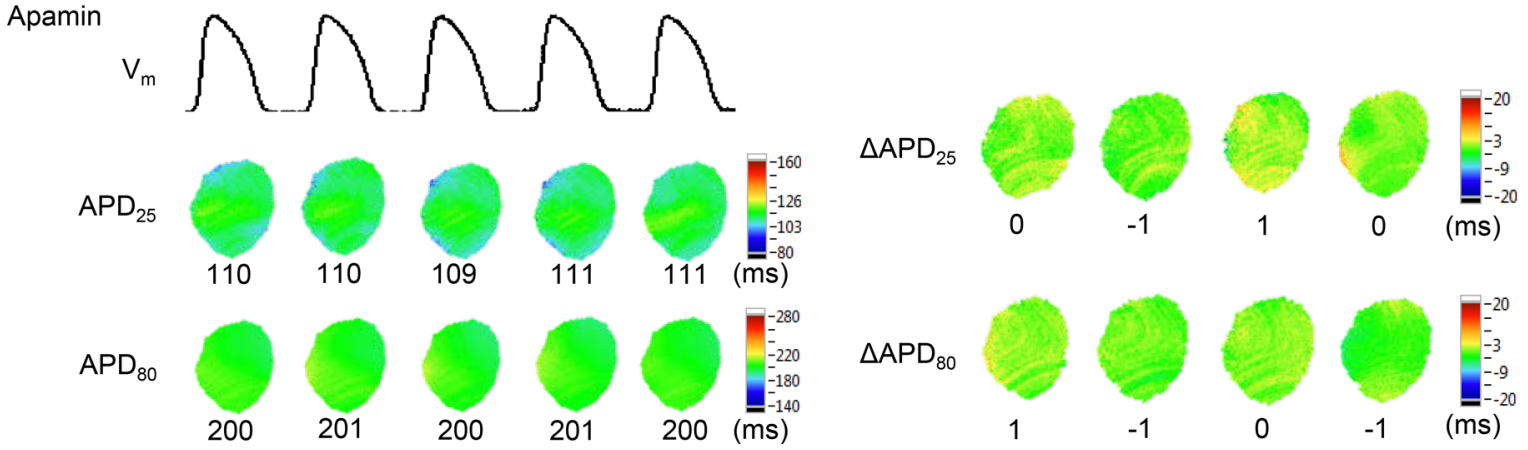

Figure 3. CyPPA induces dynamic action potential (AP) variations during sinus rhythm. (A) Representative $V_{m}$ traces, $A P D_{25}$ and $A P D_{80}$ maps of 5 continuous sinus beats at baseline, after CyPPA and after apamin (protocol I). APD at baseline was homogeneous with dynamic stability. CyPPA led to heterogeneous APD distribution with beat-to-beat AP variations. Red arrow indicates the steep APD gradient forming in the right ventricle. Subsequent apamin administration diminished the spatial heterogeneity and dynamic instability. (B) $\triangle A P D_{25}$ and $\triangle A P D_{80}$ maps show the differences between 2 successive beats. Note that dynamic APD changes were minimal at baseline and after apamin. CyPPA led to strengthened beat-to-beat APD instability.

beat 3 (Figure 5D) shows wavebreaks that degenerated into reentry in the RV. Figure 5, E-G display another episode of SVF with P2R initiation that was directly triggered by a sinus beat. The first phase singularity (PS) that was generated by wavebreaks formed in the RV. The transmembrane potential (TMP) recording in Figure $5 \mathrm{H}$ shows the development of phase 2 early after depolarization in the right ventricular outflow tract (RVOT). The onset of the AP in the RVOT occurred 17 ms prior to the QRS complex in the ECG, while $\mathrm{AP}$ onset in the LV occurred $28 \mathrm{~ms}$ after the onset of the QRS, also indicating the RV initiation. In addition, simultaneously optical mapping at 2 epicardial sites and 1 endocardial site from the RV (Figure 5I) shows that P2R was initiated between 2 epicardial sites and propagated to the endocardial site, suggesting a more important role of epicardium than endocardium in arrhythmogenicity.

The more important role of RV in arrhythmogenicity was further demonstrated in Supplemental Figure 3. The first PS of SVF and the PSs presented in the first $300 \mathrm{~ms}$ of SVF were more frequently distributed in the RV than in the LV. In addition to SVF, nonsustained ventricular tachycardia (NSVT) also commonly developed after CyPPA (Supplemental Figure 4). 
A

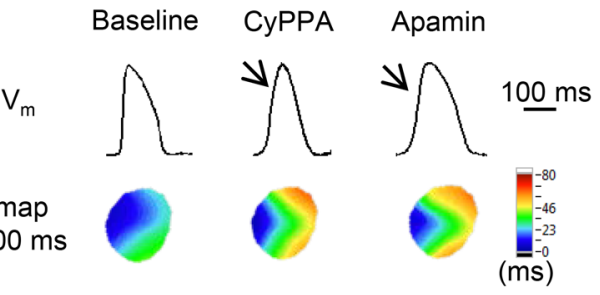

C

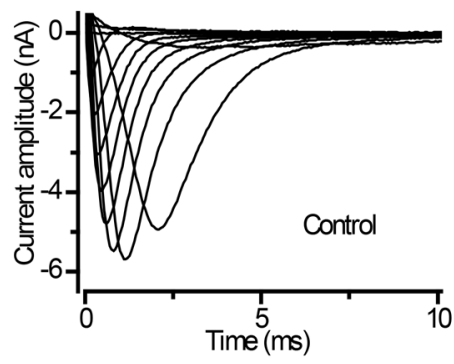

E Baseline CyPPA Apamin

$\mathrm{Ca}_{\mathrm{i}}$
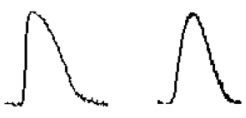

$\mathrm{Ca}_{\mathrm{i}} \mathrm{TD}_{25}$

$\mathrm{Ca}_{\mathrm{i}} \mathrm{TD}_{80}$

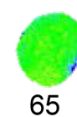

65

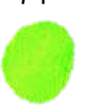

153

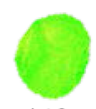

146
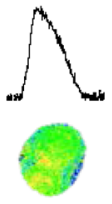

71

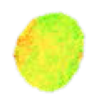

168
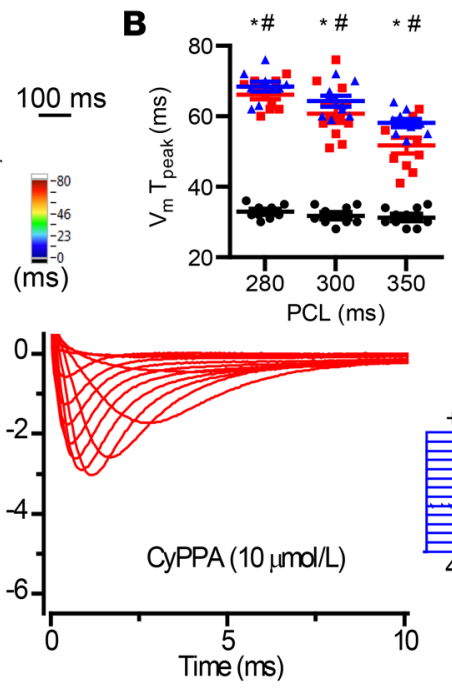

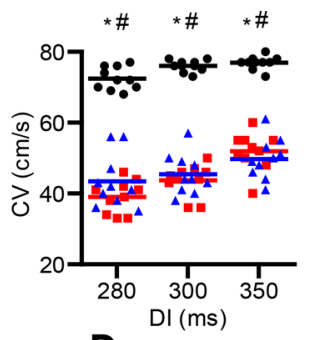

- Baseline

- CyPPA

- Apamin

* $\mathrm{p}<0.05$, Baseline vs. CyPPA $\# p<0.05$, Baseline vs. Apamin $\mathrm{N}=12$

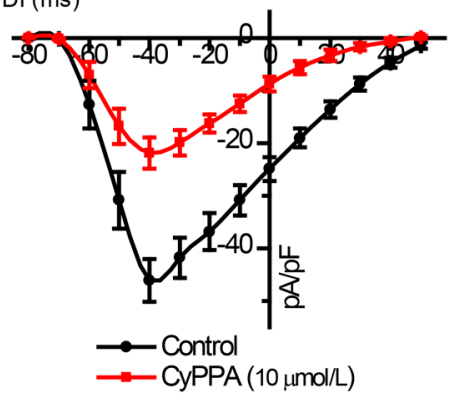

G

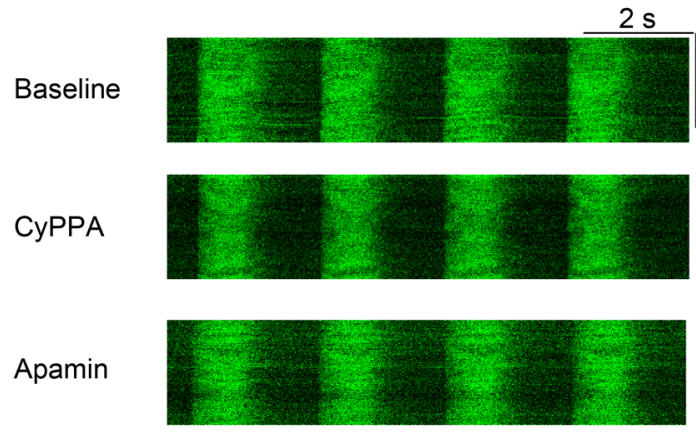

$\mathrm{s}$
F

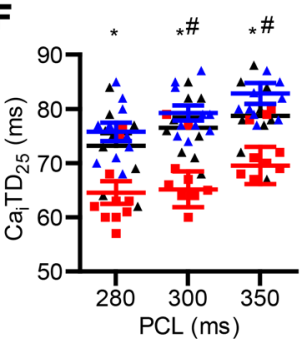

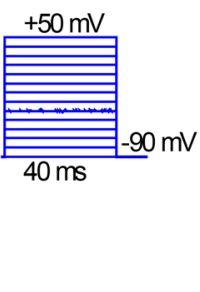

- Baseline

- CyPPA

- Apamin

$* p<0.05$, Baseline vs. CyPPA $\# p<0.05$, Apamin vs. CyPPA

$\mathrm{N}=12$
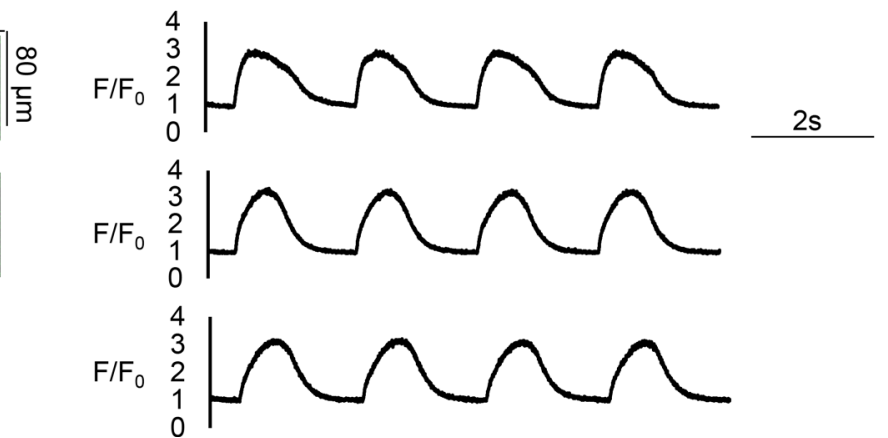

Figure 4. Effects of CyPPA and apamin on depolarization, conduction, and $\mathrm{Ca}_{\mathrm{i}}$ transient. (A) Representative $\mathrm{V}_{\mathrm{m}}$ traces and activation (AT) maps at pacing cycle length (PCL) $300 \mathrm{~ms}$ (protocol I, $n=12$ ). Compared with baseline, CyPPA decelerated action potential (AP) upstroke and intraventricular conduction velocity (CV). Subsequent apamin had little effect on AP upstroke and CV. (B) Summary of the $V_{m}$ time to peak ( $T_{\text {peak }}$ ) and CV at baseline, after CyPPA and after apamin (mean \pm SEM, 2-way ANOVA with Bonferroni's post hoc test). (C) Voltage clamp of sodium current $\left(I_{\mathrm{Na}}^{\text {peak }}\right)$ in isolated ventricular cardiomyocytes. Superimposed current traces of $I_{\mathrm{Na}}$ obtained in the absence (control) and presence of CyPPA $(10 \mu \mathrm{mol} / \mathrm{I})$. (D) Average I-V relationships of $I_{\mathrm{Na}}$ density in the absence (black) and presence of CyPPA (red) (mean \pm SEM, $n=12$ myocytes from 4 rabbits). (E) Representative $\mathrm{Ca}_{\mathrm{i}}$ traces, $\mathrm{Ca}_{\mathrm{i}}$ transient duration (Ca $\mathrm{TD}_{25}$ ) and $\mathrm{Ca}_{\mathrm{i}} \mathrm{TD}_{80}$ maps at PCL 300 ms. CyPPA abbreviated $C \mathrm{a}_{\mathrm{i}} \mathrm{TD}_{25}$ and $\mathrm{Ca}_{\mathrm{i}} \mathrm{TD}_{80}$, while apamin prolonged $\mathrm{Ca}_{\mathrm{i}} \mathrm{TD}_{25}$ and $C \mathrm{a}_{\mathrm{i}} T \mathrm{~T}_{80}$ due to slow $C \mathrm{a}_{\mathrm{i}}$ transient decay. (F) Summary of $\mathrm{Ca}_{\mathrm{i}} \mathrm{TD}_{25}$ and $\mathrm{Ca}_{\mathrm{i}} \mathrm{TD}_{80}$ at baseline, after CyPPA and after apamin (mean $\pm \mathrm{SEM}, 2$-way ANOVA with Bonferroni's post hoc test). (G) Confocal calcium imaging in fluo-4AM-loaded isolated ventricular cardiomyocytes with field stimulation at $0.5 \mathrm{~Hz}$. Left panel: original fluorescence signal. Right panel: $F / F_{0}$ traces of intracellular $\mathrm{Ca}^{2+}$ dynamics.

Activation of $S K 3$, not $S K 2$, is responsible for CyPPA-induced JWS. As a subtype-selective SK positive modulator, CyPPA at $10 \mu \mathrm{mol} / 1$ mainly activates $I_{\mathrm{KAS}}$ carried by SK3 (12). To further differentiate the effects of SK3 from SK2, rabbit hearts were pretreated with Lei-Dab 7 (15 nmol/1), a specific SK2 blocker with little effect on SK3 (16), prior to CyPPA (protocol IIA, Supplemental Figure 5). SK2 blockade by Lei-Dab7 pretreatment failed to prevent J wave elevation and SVF induced by CyPPA. However, subsequent SK3 blockade by apamin suppressed JWS and heterogeneously prolonged APD, indicating that SK3 activation is responsible for JWS. This was confirmed by the control studies in which apamin was administered prior to CyPPA (protocol IIB, 
A

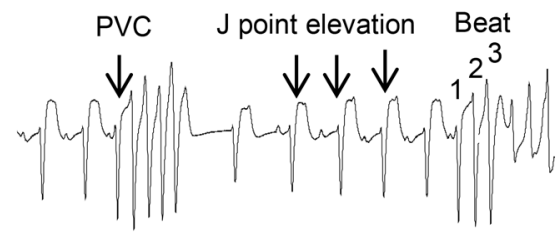

B

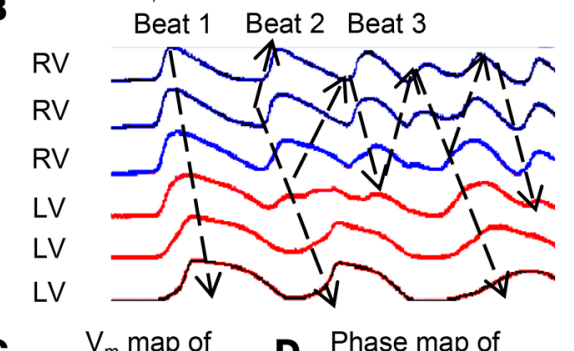

C

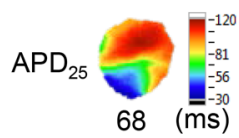

$\mathrm{APD}_{80} \underbrace{}_{165} \begin{aligned} & \bar{l}_{-200}^{-200} \\ & -157 \\ & -129 \\ & { }^{-}-100 \\ & \mathrm{~ms})\end{aligned}$
D Phase map of Beat 3

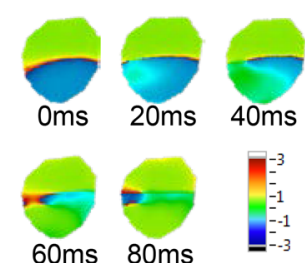

E

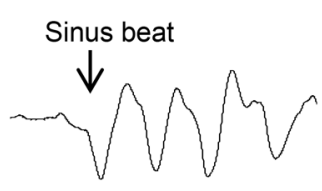

F

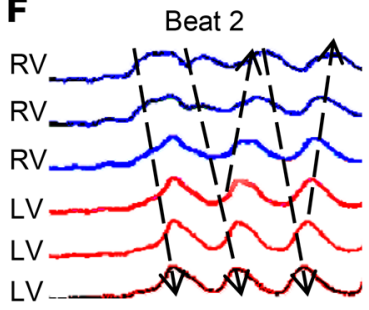

G Phase map of Beat 2

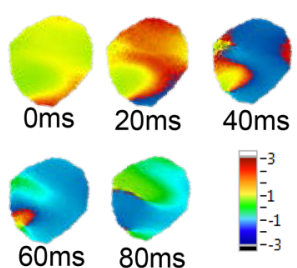

H

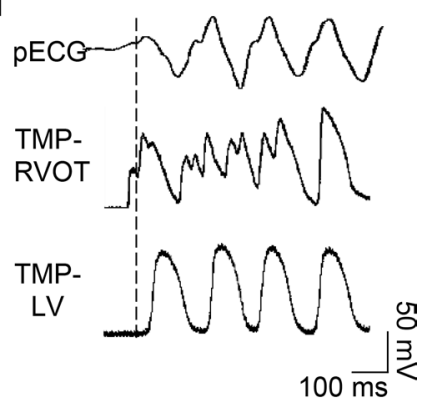

I
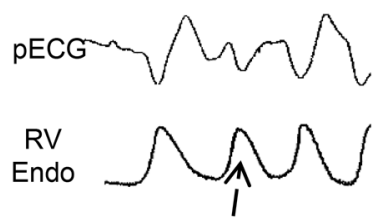

RV

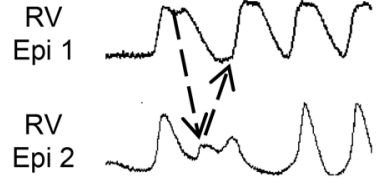

Figure 5. CyPPA induces spontaneous ventricular fibrillation (SVF) by phase $\mathbf{2}$ reentry (P2R). (A-D) Representative SVF episodes initiated by spontaneous short-coupled premature ventricular contractions (PVCs) after CyPPA. (A) pECG shows that before the onset of SVF, J wave was elevated and RR interval was gradually shortened due to type I (Wenckebach) second-degree AV block. (B) Corresponding $V_{m}$ traces shows P2R. (C) $V_{m} m a p$ of beat 1 exhibits steep action potential duration (APD) gradient prior to the onset of SVF. (D) Phase map of beat 3 shows wavebreaks that degenerated into reentry. (E-G) An episode of SVF was directly initiated from a sinus beat after CyPPA. (E) pECG. (F) Corresponding $V_{m}$ map shows P2R. (G) Phase map of beat 2 shows wavebreaks which degenerated into reentry in the RV. (H) Simultaneous ECG and transmembrane potential (TMP) recordings at the onset of SVF. The TMP recorded from a ventricular cardiomyocyte at RVOT exhibits phase 2 early afterdepolarization. The action potential onset in the right ventricular outflow tract (RVOT) occurred 17 ms prior to the QRS complex, while action potential onset in the left ventricle (LV) occurred 28 ms after the onset of QRS complex. Black vertical line indicates the QRS onset. (I) Simultaneous optical traces at 2 epicardial sites and 1 endocardial site from the RV at the onset of SVF. P2R was generated between 2 RV epicardial sites and propagated to the endocardial site, thus precipitating SVF.

Supplemental Figure 6). With apamin pretreatment blocking both SK2 and SK3, CyPPA failed to induce J wave elevation. Although PVCs were still frequently observed, none of them degenerated into SVF. In the presence of apamin, CyPPA prolonged APD due to delayed AP upstroke by $I_{\mathrm{Na}}$ blockade.

Sex and regional differences. As shown in Supplemental Figure 7, males had significantly higher CyPPAinduced $\mathrm{J}$ wave elevation ( $\triangle \mathrm{J}$ point amplitude: $0.27 \pm 0.07 \mathrm{mV}$ vs. $0.21 \pm 0.02 \mathrm{mV}, P<0.05$ ) and more episodes of SVF/SVT (16 \pm 8 vs. $5 \pm 1, P<0.001$ ) than females. Electrical storm (ES) only developed in males. However, the differences in $\triangle \mathrm{APD}$ were insignificant between sexes. The differences in the quantitative PCR (qPCR) measurements of SK3 mRNA expression (normalized to GAPDH, $\times 100$ ) among tissue preparation of male RVOT, male LV base (LVB), female RVOT, and female LVB were also insignificant.

Therapeutic approaches for CyPPA-induced JWS. As shown above, $I_{\mathrm{KAS}}$ blockade by apamin is antiarrhythmic in CyPPA-treated rabbit hearts. Supplemental Figure 8 exhibits another typical case of ES induced by CyPPA in a male rabbit. SVF was originated from RVOT (site 1) and the reentry traveled along the path of 1-4-5-2-1. In contrast, ES was terminated after apamin despite frequent spontaneous PVCs. The PVC was originated from the similar region at RVOT (site 1) but failed to complete the reentry.

Since isoproterenol suppresses ES in BrS clinically (17), we tested the effects of isoproterenol on CyPPA-induced JWS (protocol IV, $n=6$ ). Isoproterenol eliminated SVF in all 6 hearts. As shown in Figure 6A, $\mathrm{J}$ wave was accentuated and an episode of SVF developed after CyPPA. A total of 8 shocks were applied to attempt to defibrillate. The first 7 shocks failed according to ECG, but the eighth attempt succeeded. After isoproterenol, J wave was slightly attenuated and no SVF developed. Two episodes of VF were induced by ventricular pacing. The first episode was successfully defibrillated by shock 9 , while the second episode was self-terminated. Figure $6 \mathrm{~B}$ shows that during sinus rhythm after CyPPA, both $\mathrm{APD}_{25}$ and $\mathrm{APD}_{80}$ exhibited heterogeneous dispersions with large gradients, especially in the RV. The activation map displayed impaired intraventricular conduction. After isoproterenol, $\mathrm{APD}_{25}$ and $\mathrm{APD}_{80}$ were markedly shortened but became 


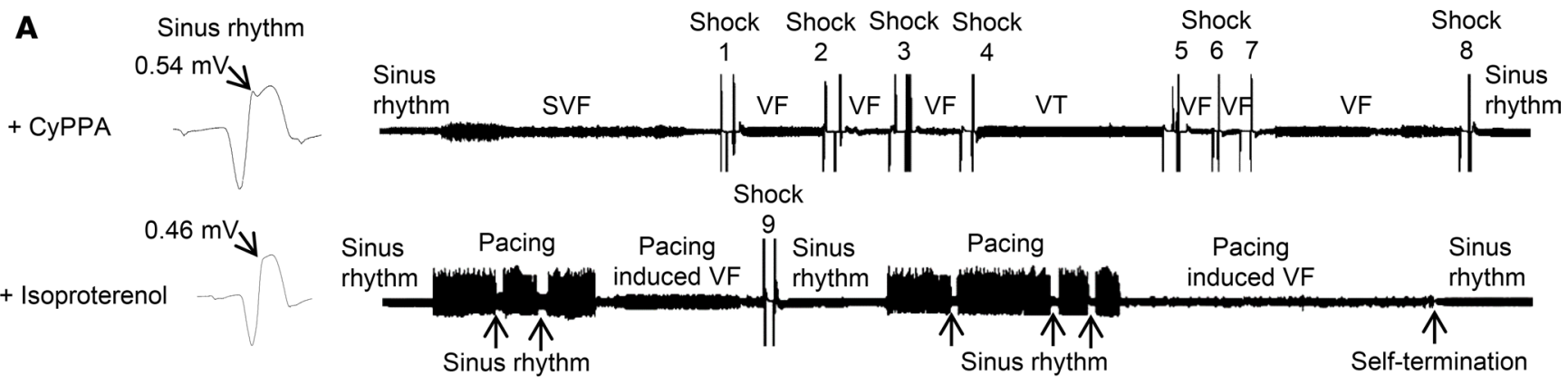

B

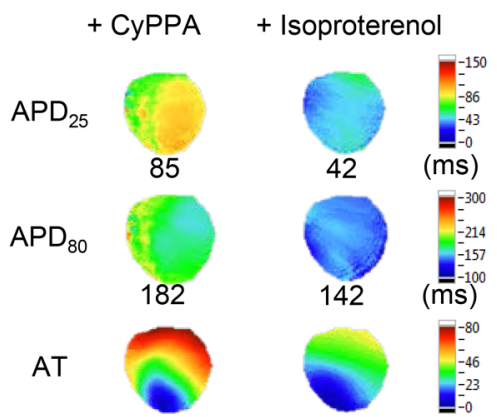

C $\mathrm{Ca}_{\mathrm{i}}$

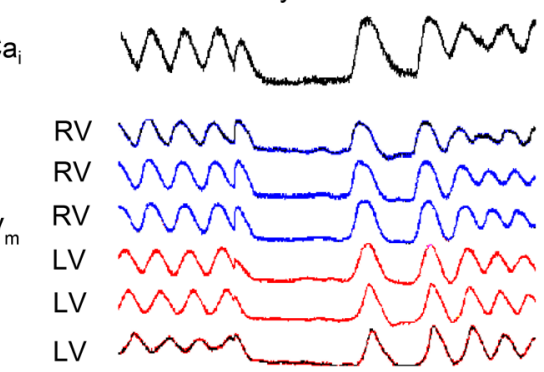

+ Isoproterenol

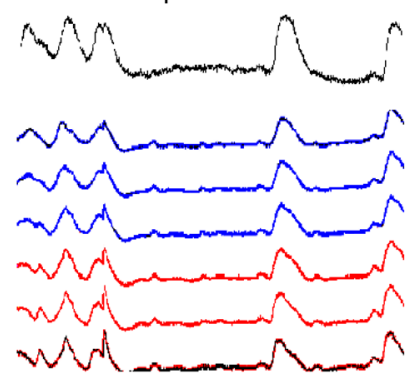

Figure 6. Isoproterenol is antiarrhythmic in CyPPA-induced J wave syndrome. Protocol III $(n=6)$. (A) pECG after CyPPA (upper panel) shows J wave elevation during sinus rhythm and an episode of spontaneous ventricular fibrillation (SVF). The SVF was shock resistant (shocks 1-7), which was defibrillated by the eighth attempt of shock (shock 8). After isoproterenol infusion (lower panel), J wave was slightly attenuated and no SVF was generated. Ventricular pacing induced 2 episodes of VF. The first episode was defibrillated by the first attempt (shock 9 ). The second episode was self-terminated. (B) Optical maps during sinus rhythm. During CyPPA, action potential duration $\left(\mathrm{APD}_{25}\right)$ and $A P D_{80}$ maps exhibited heterogeneous $A P D$ distribution and large APD gradient, especially in the right ventricle. The activation (AT) map shows slow intraventricular conduction. Subsequent isoproterenol markedly shortened $A \mathrm{PD}_{25}$ and $A \mathrm{PD}_{80}$, diminished $\mathrm{APD}$ heterogeneities, and accelerated conduction velocity. (C) Simultaneous optical traces of $\mathrm{Ca}_{\mathrm{i}}$ and $\mathrm{V}_{\mathrm{m}}$ at the time of defibrillation. During CyPPA, the shock-resistant VF on ECG was actually successfully defibrillated by shock 1 . However, phase 2 reentry was immediately reinitiated after 1 sinus beat, such that VF appeared to sustain on pECG. After isoproterenol, the pacing-induced VF was successfully defibrillated by the first attempt (shock 9 ) without reinitiation.

more homogeneous with accelerated conduction velocity. Figure 6C shows optical traces at the time of defibrillation. During CyPPA, the apparent shock-resistant VF on ECG was actually successfully defibrillated. However, P2R was immediately re-initiated after only 1 sinus beat, such that VF seemed sustained on ECG. After isoproterenol, the pacing-induced VF was successfully defibrillated without reinitiation. These results indicate that isoproterenol is antiarrhythmic in CyPPA-induced JWS.

$I_{K A S}$ activation induces Osborn waves and phase 2 repolarization alternans during hypothermia. Environmental factors can also lead to the appearance of ECG patterns and arrhythmias similar to JWS in the absence of any apparent genetic abnormality. Among a variety of acquired conditions, classical J waves are induced by hypothermia, also known as Osborn waves. As hypothermia reduced $I_{\mathrm{Na}}(3,4)$, we hypothesized that $I_{\mathrm{KAS}}$ is concomitant activated during hypothermia, and thus contributes to the generation of Osborn waves. We created hypothermia $\left(32.0^{\circ} \mathrm{C}\right)$, followed by apamin administration (at $32.0^{\circ} \mathrm{C}$ ) and rewarming $\left(38.3^{\circ} \mathrm{C}\right.$ ) in 6 hearts (optical mapping, protocol IV). Osborn waves were induced in 5 hearts and were attenuated by subsequent apamin in 3 hearts. SVF was developed in 2 hearts during hypothermia (1 episode for each), while no SVF was observed after apamin (Figure 7, $\mathrm{A}$ and $\mathrm{B})$. The results from ex vivo $\mathrm{pECG}$ recordings were verified by in vivo surface ECG studies in 2 rabbits (Supplemental Figure 10), which also showed that $I_{\text {KAS }}$ inhibition by apamin suppressed Osborn waves in both rabbits.

Optical mapping data in Figure 7C show that, compared with baseline, APD prolonged during hypothermia, which might be correlated with the increased $\mathrm{Ca}_{\mathrm{i}}$ transient. $I_{\mathrm{KAS}}$ blockade by apamin further prolonged APD more prominently at $\mathrm{APD}_{25}$ than at $\mathrm{APD}_{80}$, suggesting $I_{\mathrm{KAS}}$ activation at early repolarization phases. After rewarming, $\mathrm{Ca}_{\mathrm{i}} \mathrm{TD}$ and $\mathrm{APD}$ were shortened towards baseline. We further performed rapid ventricular pacing to induce $\mathrm{Ca}_{\mathrm{i}}$ and $\mathrm{V}_{\mathrm{m}}$ alternans. As shown in Supplemental Figure 9, at baseline with normothermia, $\mathrm{Ca}_{\mathrm{i}}$ and $\mathrm{V}_{\mathrm{m}}$ were positively coupled and exhibited spatial concordance. However, during hypothermia (Figure 7D), $\mathrm{Ca}_{\mathrm{i}}$ and $\mathrm{V}_{\mathrm{m}}$ became negatively coupled (electromechanically discordant) at 
A $\begin{array}{cccc}\text { Baseline } & \text { Hypothermia } & \text { Apamin } \\ 38.3^{\circ} \mathrm{C} & 32.0^{\circ} \mathrm{C} & \text { Rewarming } \\ 38.3^{\circ} \mathrm{C}\end{array}$

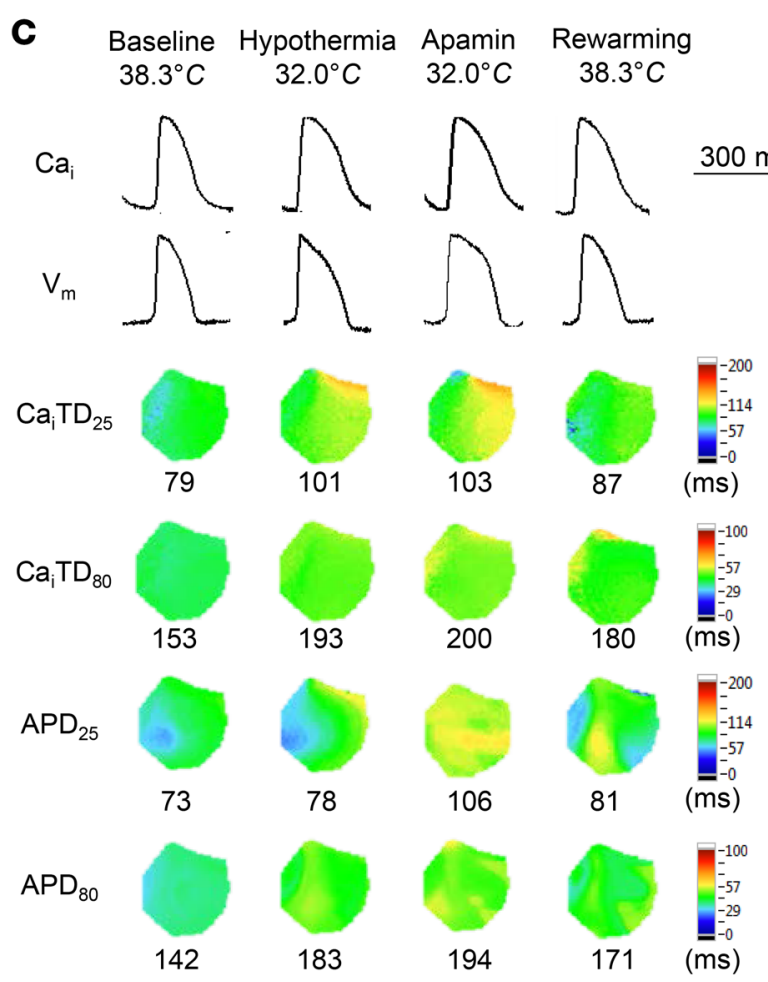

B
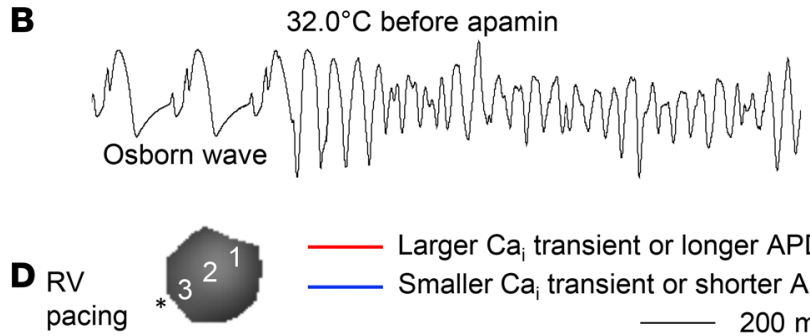

Larger $\mathrm{Ca}_{\mathrm{i}}$ transient or longer $\mathrm{APD}_{80}$ Smaller $\mathrm{Ca}_{\mathrm{i}}$ transient or shorter $\mathrm{APD}_{80}$ $200 \mathrm{~ms}$
Before apamin $\left(32.0^{\circ} \mathrm{C}\right)$ Beat 1 Beat 2

$\mathrm{Ca}$

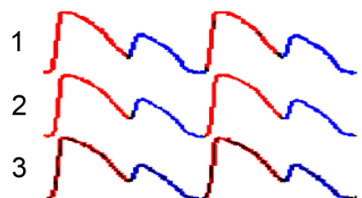
$1 N \Omega N$ $\mathrm{V}_{\mathrm{m}}$

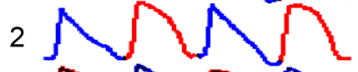
$3 \sqrt[N]{ }$ Beat Beat Beat2 $\begin{array}{lll}1 & 2 & \text {-Beat1 }\end{array}$ $\mathrm{APD}_{25}$<smiles>c1ccccc1</smiles>
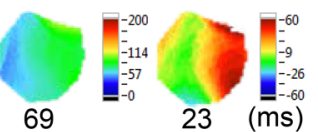

$\mathrm{APD}_{80}$
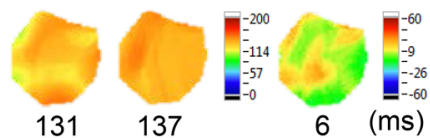

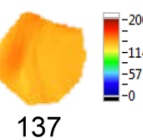

After apamin $\left(32.0^{\circ} \mathrm{C}\right)$

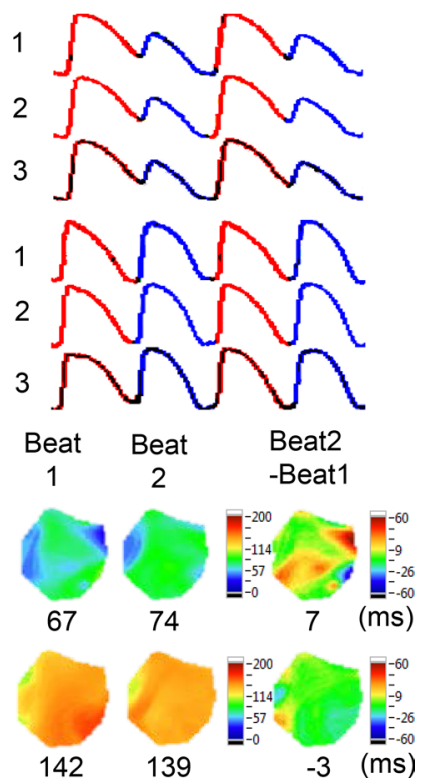

Beat 1 Beat 2

Figure 7. $I_{\text {KAS }}$ activation induces Osborn waves and phase 2 repolarization alternans during hypothermia. Protocol IV $(n=6)$. (A) ECC of sinus rhythm shows Osborn waves during hypothermia, which was attenuated by apamin. (B) During hypothermia before apamin, an episode of spontaneous ventricular fibrillation was triggered by a spontaneous short-coupled premature ventricular contraction. (C) Optical maps of $\mathrm{Ca}_{\text {a }}$ and $\mathrm{V}_{\mathrm{m}}$ at pacing cycle length $300 \mathrm{~ms}$. Compared with baseline, hypothermia prolonged both $\mathrm{Ca}_{\mathrm{i}}$ transient duration ( $\left.\mathrm{Ca}_{\mathrm{i}} \mathrm{TD}\right)$ and action potential duration (APD). Apamin had limited effect on CaTD, but further prolonged $A P D$ more prominently at $A \mathrm{PD}_{25}$ than at $A P D_{80}$. After rewarming, $\mathrm{Ca}_{\mathrm{i}} \mathrm{TD}$ and $\mathrm{APD}$ were shortened towards baseline. (D) During hypothermia, $C a_{\mathrm{i}}$ and $V_{m}$ alternans were induced by ventricular pacing at $200 \mathrm{~ms}$. Before apamin, $\mathrm{Ca}_{\mathrm{i}}$ and $\mathrm{V}_{\mathrm{m}}$ were negatively coupled at distal sites (site 2 and 3 ) but remained positive coupling at proximal sites (site 1 ), leading to spatially discordant $V_{m}$ alternans. The difference between 2 consecutive beats (beat 2 - beat 1 ) was larger at $A P D_{25}$ than at $A P D_{80}$ (phase 2 repolarization alternans). Apamin differentially prolonged $A P D$ at $A P D_{25}$ and $A P D_{80}$ and in beat 1 and beat 2 . The prolongation was more prominent in beat 1 , which coupled with larger $\mathrm{Ca}_{i}$ transient than in beat 2 coupled with smaller $\mathrm{Ca}_{\mathrm{i}}$ transient, and more prominent at $\mathrm{APD}_{25}$ when $\mathrm{Ca}_{\mathrm{i}}$ was more abundant than at $\mathrm{APD}_{80}$. As a result, apamin eliminated the negative $\mathrm{Ca}_{\mathrm{i}}-\mathrm{V}_{\mathrm{m}}$ coupling and attenuated phase 2 repolarization alternans.

distal sites (site 2 and 3 ) but remained positively coupled at the proximal site (site 1), leading to spatially discordant $\mathrm{V}_{\mathrm{m}}$ alternans. The difference between 2 consecutive beats (beat 2 - beat 1) was larger at $\mathrm{APD}_{25}$ than at $\mathrm{APD}_{80}$, indicating alternans was more prominent at early repolarization phases. $I_{\mathrm{KAS}}$ blockade by apamin differentially prolonged $\mathrm{APD}$ at $\mathrm{APD}_{25}$ and $\mathrm{APD}_{80}$ and in beat 1 and beat 2 . The prolongation was more prominent at beat 1 coupled to the larger $\mathrm{Ca}_{\mathrm{i}}$ transient than at beat 2 coupled to the smaller $\mathrm{Ca}_{\mathrm{i}}$ transient, and was more prominent at $\mathrm{APD}_{25}$ when $\mathrm{Ca}_{1}$ was higher than that at $\mathrm{APD}_{80}$. As a result, negative $\mathrm{Ca}_{\mathrm{i}}-\mathrm{V}_{\mathrm{m}}$ coupling was eliminated and phase 2 alternans was attenuated. Taken together, our results show that $I_{\text {KAS }}$ is activated during hypothermia and participates in the generation of Osborn waves. $I_{\text {KAS }}$ blockade may serve as a potential therapeutic approach for preventing ventricular arrhythmia during hypothermia.

\section{Discussion}

$I_{\mathrm{KAS}}$ activation with concomitant $I_{\mathrm{Na}}$ inhibition by CyPPA recapitulates JWS in Langendorff-perfused rabbit hearts. CyPPA also causes sinus bradycardia, atrial tachyarrhythmias, AV block, and intraventricular conduction delay, which have been observed in BrS (18). We summarized the manifestations of clinical BrS, ERS, and the CyPPA-induced JWS models in Supplemental Table 2. There are significant similarities among them. 
CyPPA and SK3 activation. CyPPA is the first subtype-selective potentiator of SK3 channels (SK3 > SK2 >>> SK1) (12). It does not activate $\mathrm{Ca}^{2+}$ and other voltage-dependent $\mathrm{K}^{+}$currents; however, it causes an inhibition of $\mathrm{BK}$ and $\mathrm{Na}^{+}$currents (12). CyPPA binds to and allosterically modulates via a binding pocket situated at the intracellular interface of SK and calmodulin, which increases the SK/calmodulin's affinity for $\mathrm{Ca}^{2+}(19)$. Due to overlapping activation ranges between SK3 $\left(\mathrm{EC}_{50}=5.6 \pm 1.6 \mu \mathrm{mol} / \mathrm{l}\right)$ and SK2 $\left(\mathrm{EC}_{50}=14 \pm 4 \mu \mathrm{mol} / \mathrm{l}\right)$ and the possibility of forming heteromeric complexes $(20,21)$, whether CyPPA at the current concentration can activate heteromeric channels remains unclear. However, SK2 and SK3 activation might lead to different manifestations. While overexpression of SK2 resulted in tachycardia and increased AV node conduction (22), overexpression of SK3 led to bradycardia, conduction disturbances, and ventricular APD dispersion, resulting in sudden cardiac death $(11,23)$. The latter finding mimicked the phenotypes observed in this CyPPA model of $\mathrm{BrS}$. The dominance of SK3 activation was also supported by the ability for CyPPA to induce JWS after pretreatment with SK2-specific blocker Lei-Dab 7, but prevention of JWS after treatment with apamin (Supplemental Figures 5 and 6). Therefore, we propose that the development of JWS is mainly attributed to SK3 rather than SK2 activation. Because CyPPA exclusively increases the $\mathrm{Ca}^{2+}$ sensitivity of SK3 activation without effect on maximally activated channels (12), the results of this study suggest that modulation of SK3 channel $\mathrm{Ca}^{2+}$ sensitivity could be an endogenous mechanism for fine-tuning cardiac repolarization and arrhythmogenesis.

$I_{K A S}$ activation and arrhythmogenesis. $I_{\mathrm{KAS}}$ is minimally activated in normal rabbit ventricles and is upregulated and antiarrhythmic when repolarization reserve is impaired, such as in heart failure and hypokalemia $(24,25)$. Because normal rabbit hearts already exhibit abundant repolarization reserve, as we proposed here, with concomitant $I_{\mathrm{Na}}$ inhibition, excessive $I_{\mathrm{KAS}}$ activation in normal hearts is highly arrhythmogenic by inducing early and heterogeneous repolarization, especially in the RV epicardium. These findings are consistent with the heterogeneity of repolarization within the RVOT epicardium in patients of BrS receiving pilsicainide challenge (26). Since CyPPA increases $I_{\mathrm{KAS}}$ sensitivities to $\mathrm{Ca}^{2+}$, in some regions, the excessive activation of $I_{\mathrm{KAS}}$ counterbalances the APD-prolonging effects of $\mathrm{Ca}^{2+}$ influx, resulting in the loss of AP domes and early repolarization. In other regions, however, $I_{\mathrm{KAS}}$ activation drives the membrane voltage into the window with delayed $I_{\mathrm{Ca}, \mathrm{L}}$ inactivation. The enlarged calcium window current overwhelms the outward currents, leading to a prominent AP dome and prolonged APD. The electrophysiological heterogeneity creates a large electrical gradient between regions, thus facilitating $\mathrm{P} 2 \mathrm{R}$. P2R related to $I_{\text {to }}$ is thought to originate primarily from the epicardium (27), which is in line with our observations in Figure 5D with $I_{\text {KAS }}$ activation. However, since epicardium expresses significantly more $I_{\mathrm{KAS}}$ than the mid-myocardium layer in both human and rabbit ventricles $(6,28)$, the transmural heterogeneity of $I_{\text {KAS }}$ might also contribute to the arrhythmogenesis.

A previous experimental study in canines showed that enhanced $I_{\text {to }}$ caused APD alternans (29), which was later confirmed by computer simulations (30). $I_{\text {to }}$-mediated alternans or other complex AP dynamics (such as chaos) are associated with unstable switching AP morphology even in homogeneous cables, which underlies P2R formation (31). In the present study, irregular alternans or chaos (Figure 3) is potentiated by $I_{\text {KAS }}$ activation, suggesting that $I_{\text {KAS }}$ could act like $I_{\text {to }}$ in AP dynamics. In addition to spatial heterogeneity due to different anatomical properties or fibrosis in RVOT (15), our data suggest that dynamic instability is also important in the arrhythmogenesis of JWS.

$I_{\text {KAS }}$ is an important coupler between $\mathrm{Ca}_{\mathrm{i}}$ and $\mathrm{V}_{\mathrm{m}}$ due to its $\mathrm{Ca}^{2+}$ sensitivity (32). $\mathrm{Ca}_{\mathrm{i}}$ and $\mathrm{V}_{\mathrm{m}}$ are positively coupled (i.e., a large $\mathrm{Ca}_{\mathrm{i}}$ transient prolongs and a small $\mathrm{Ca}_{\mathrm{i}}$ transient shortens APD) in the absence of $I_{\mathrm{KAS}}(32)$. Mathematically introducing $I_{\mathrm{KAS}}$ could shorten APD when $\mathrm{Ca}_{\mathrm{i}}$ transient is large, which results in negative $\mathrm{Ca}_{\mathrm{i}}-\mathrm{V}_{\mathrm{m}}$ coupling (32). In this study, we experimentally validated this prediction in the hypothermia model. During hypothermia, the decreased peak $I_{\mathrm{Ca}, \mathrm{L}}$ reduces $\mathrm{Ca}^{2+}$-dependent $I_{\mathrm{Ca}, \mathrm{L}}$ inactivation and prolongs $\mathrm{Ca}_{\mathrm{i}}$ transient, which in turn increases sarcoplasmic $\mathrm{Ca}^{2+}$ stores and release (33). $I_{\mathrm{KAS}}$ is more abundantly activated at early repolarization phases and at the beat with larger $\mathrm{Ca}_{\mathrm{i}}$ transient, leading to faster phase 2 repolarization (shorter $\mathrm{APD}_{25}$ and AP triangulation), while $I_{\mathrm{KAS}}$ is less abundantly activated at the beat with smaller $\mathrm{Ca}_{\mathrm{i}}$ transient, leading to slower phase 2 repolarization (longer $\mathrm{APD}_{25}$ and $\mathrm{AP}$ squaring). In this situation, the coupling between $\mathrm{Ca}_{1}$ transient and $\mathrm{APD}_{25}$ turns into negative, resulting in phase 2 repolarization alternans with electromechanical discordance, especially at regions distal to the pacing site that harbor enriched $\mathrm{Ca}^{2+}$ accumulation and $I_{\mathrm{KAS}}$ activation (24). $I_{\mathrm{KAS}}$ blockade by apamin prominently prolongs APD at the beats with large $I_{\mathrm{KAS}}$ activation, at early repolarization phase, and at regions distal to the pacing site, and thus attenuates negative $\mathrm{Ca}_{\mathrm{i}}-\mathrm{V}_{\mathrm{m}}$ coupling at early repolarization phase and its spatial discordance. We propose that phase 2 repolarization alternans facilitates $\mathrm{P} 2 \mathrm{R}$. $I_{\mathrm{KAS}}$ blockade prevents hypothermic ventricular arrhythmias by the attenuation of the spatially and electromechanically discordant phase 2 repolarization alternans. 
Clinically, bradycardia and conduction delay are commonly observed in BrS (15). In this CyPPA model, AV node and intraventricular conduction are also impaired. Since CyPPA at $10 \mu \mathrm{mol} / 1$ reduces peak $I_{\mathrm{Na}}$ by approximately $60 \%$ in ventricular myocytes, these effects on cardiac conduction as well as the decreased AP upstroke velocity are attributed, at least partially, to $I_{\mathrm{Na}}$ blockade. However, the slow conduction seems large given the degree of $I_{\mathrm{Na}}$ blockade. One possible explanation is that large APD heterogeneity leads to heterogeneity of refractory period, which impairs conduction velocity (postrepolarization refractoriness). A second reason is that SK channels are abundantly present in intercalated discs (28). Mice with SK3 overexpression develop conduction block (11), suggesting that $I_{\mathrm{KAS}}$ may directly participate in the cardiac conduction. Additionally, conduction does not only rely on the coupling between myocytes, but also on the Purkinje system and the coupling between Purkinje cells and cardiomyocytes. It is possible that the CyPPA reduces $I_{\mathrm{Na}}$ more in Purkinje cells than ventricular cardiomyocytes. Finally, $I_{\mathrm{KAS}}$ density is larger in the Purkinje system than cardiomyocytes (34). It is possible that slow conduction is caused by $I_{\mathrm{Na}}$ inhibition and $I_{\text {KAS }}$ activation in the His-Purkinje system. We also observed slow upstroke of $\mathrm{Ca}^{2+}$ transient induced by CyPPA (Figure 4E). Since CyPPA blocks $I_{\mathrm{Na}}$, the slow upstroke of $\mathrm{Ca}^{2+}$ transient is probably secondary to the slow depolarization. The slow depolarization leads to decreased activation of L-type $\mathrm{Ca}^{2+}$ current, which further results in insufficient $\mathrm{Ca}^{2+}$-induced $\mathrm{Ca}^{2+}$ release from the sarcoplasmic reticulum. The slow $\mathrm{Ca}^{2+}$ upstroke was also observed in the induced pluripotent stem cell-derived cardiomyocytes from $\mathrm{BrS}$ patients with SCN5A mutation (35). Since depolarization and repolarization are never independent, the $I_{\mathrm{KAs}}$-mediated concomitant effects of early repolarization and dysfunction of depolarization and conduction might reveal the potential mechanisms contributed to JWS.

Genetic basis and acquired conditions. JWS are considered genetic diseases. BrS and ERS have been associated with variants in 18 and 7 genes, respectively (1). However, these genetic variants accounted for only one third of JWS patients (1). It is possible that gain-of-function mutations in KCNN3 could directly increase $I_{\mathrm{KAS}}$ and facilitate the development of JWS. Single-nucleotide polymorphisms and other variants in $K C N N 3$ should be evaluated in future genetic scanning. In addition, $I_{\mathrm{KAS}}$ activation by CyPPA and its affinity for $\mathrm{Ca}^{2+}$ are regulated by a number of coassembled modulator proteins, including calmodulin, casein kinase 2, and phosphatidylinositol bisphosphate (36). Genetic variants in these SK modulators may promote the development of JWS. More importantly, in BrS patients who carry SCN5A or other sodium channel loss-of-function mutations, the activation of $I_{\text {KAS }}$ by drugs or other environmental factors might largely increase their vulnerabilities to lethal cardiac arrhythmias. On the other hand, we also demonstrated the contribution of $I_{\mathrm{KAS}}$ activation to hypothermic $\mathrm{J}$ wave and proarrhythmia. Taken together, the results of our study suggest that $I_{\text {KAS }}$ might play important roles in either genetic or acquired JWS.

Therapeutic approaches. By prolonging early repolarization phases and reducing AP dispersion and dynamic instability, apamin suppresses J wave and SVF, suggesting $I_{\mathrm{KAS}}$ blockade as a potential therapeutic approach for JWS. Unfortunately, apamin cannot be used clinically due to its neurotoxicity. AP14145, a newly synthesized $I_{\mathrm{KAS}}$ negative modulator (37), might be a promising candidate for future use in JWS. Amiodarone suppresses $I_{\mathrm{KAS}}$ at its therapeutic concentration (10-20 $\left.\mu \mathrm{mol} / 1\right)(38)$. However, it has $I_{\mathrm{Na}}$ blocking properties. Recently, we showed that ondansetron is an $I_{\mathrm{KAS}}$ blocker (39). Whether or not it is useful in managing JWS remains unknown. Further research to develop cardioselective and SK3 subtype-specific $I_{\text {KAS }}$ blockers may have promise as an antiarrhythmic strategy in JWS.

The $\beta$-adrenergic agonist isoproterenol has a class IIa recommendation for BrS patients presenting with recurrent ES (1). In this study, isoproterenol ameliorated CyPPA-induced ES. Since isoproterenol augments $I_{\mathrm{Ca}, \mathrm{L}}$ and subsequent RyR $\mathrm{Ca}^{2+}$ release, isoproterenol alone would activate $I_{\mathrm{KAS}}$. In comparison, CyPPA increases the sensitivity of SK channel to $\mathrm{Ca}^{2+}$ without increasing the maximal activated $I_{\mathrm{KAS}}$ conducted by SK3. Notably, CyPPA even induces a small reduction of the current conducted by SK2 at high $\mathrm{Ca}^{2+}$ concentrations. It is possible that $I_{\mathrm{KAS}}$ is already abundantly activated by CyPPA, especially at early repolarization phases when $\mathrm{Ca}^{2+}$ is high. Therefore, with CyPPA pretreatment, $I_{\mathrm{KAS}}$ might respond weakly to the further increase of $\mathrm{Ca}^{2+}$. In contrast, isoproterenol increases and rebalances multiple $\mathrm{Ca}^{2+}$ currents such as $I_{\mathrm{Ks}}$ and chloride currents, which may explain the further shortening of APD. Isoproterenol also augments $I_{\mathrm{Na}}(40)$, and thus increases the conduction velocity and decreases the heterogeneity of action potential. Both of these actions may be antiarrhythmic.

Limitations. While we showed that CyPPA could induce JWS in rabbits, whether or not $I_{\text {KAS }}$ activation can cause human JWS remains unclear. Due to the very limited availability of human heart tissue, especially with $\mathrm{BrS}$, it is challenging to directly perform experiments in humans. However, the rabbit model 
is considered a good animal model for studying cardiac ion currents relevant to humans (41). Because we were not able to measure the absolute values of the action potential, it is unclear if $I_{\text {KAs }}$ activation changed the resting membrane potentials of the hearts studied. However, given the high inward rectifier $\mathrm{K}^{+}$conductance due to $I_{\mathrm{K} 1}$ in rabbit ventricles, it is unlikely that $I_{\mathrm{KAS}}$ during diastole would significantly affect resting potential. The Langendorff-perfused hearts do not have intact autonomic innervation. Therefore, we are not able to determine the importance of the autonomic nervous system in the arrhythmogenesis in JWS.

\section{Methods}

Animals. A total of 52 adult (5-6 months old at the time of euthanasia) New Zealand White rabbits (28 males) with body weight 2.7 to $3.5 \mathrm{~kg}$ were used in this study. Among them, 32 (18 males) were used for optical mapping, 4 (2 males) were used for TMP recording, 10 (5 males) were used for qPCR, 4 (2 males) were used for ventricular cardiomyocytes isolation followed by patch clamp study and confocal calcium imaging, and 2 (1 male) for in vivo surface ECG recording.

Ex vivo studies. Rabbits were euthanized by i.v. sodium pentobarbitone overdose (160 mg/kg, i.v.). Hearts were harvested and Langendorff perfused with Tyrode's solution (in mmol/1: $128.3 \mathrm{NaCl}, 4.7 \mathrm{KCl}$, $20.2 \mathrm{NaHCO}_{3}, 0.4 \mathrm{NaH}_{2} \mathrm{PO}_{4}, 1.8 \mathrm{CaCl}_{2}, 1.2 \mathrm{MgSO}_{4}, 11.1$ glucose, and $40 \mathrm{mg} / 1$ bovine serum albumin [BSA]) that was bubbled with $95 \% \mathrm{O}_{2} / 5 \% \mathrm{CO}_{2}$ to maintain a $\mathrm{pH}$ of 7.40 . All chemicals were purchased from Sigma-Aldrich.

pECG recording. pECG was simultaneously recorded during the optical mapping and the TMP recording. In the tissue bath, 2 electrodes were placed close to the right atrium (RA) and the apex of LV, respectively, while the third electrode was used as ground (Supplemental Figure 11A). The signals were filtered with a bandpass from $0.5 \mathrm{~Hz}$ to $150 \mathrm{~Hz}$ with sampling rate $1,000 \mathrm{~Hz}$. A representative pECG at baseline is shown in Supplemental Figure 11B. We defined the terms of pECG morphology as follows (Supplemental Figure 11C):

$J$ wave: an elevation of the QRS-ST junction exceeding $0.1 \mathrm{mV}$ above the baseline level, including Brugada wave and ER wave.

Type 1 Brugada wave: a coved-type ST elevation greater than $0.2 \mathrm{mV}$ followed by a gradually descending ST segment.

Type 2 Brugada wave: a saddle-back ST elevation greater than $0.2 \mathrm{mV}$ followed by a convex ST greater than $0.1 \mathrm{mV}$.

$E R$ : J wave greater than $0.1 \mathrm{mV}$, excluding type 1 and type 2 Brugada waves.

In addition, JWS was defined as a combination of J wave and the development of SVF or SVT. ES was defined as at least 10 recurrent episodes of SVF/SVT during the experiment.

Optical mapping. Simultaneous optical mapping of $\mathrm{Ca}_{\mathrm{i}}$ and $\mathrm{V}_{\mathrm{m}}$ was performed using techniques similar to that reported elsewhere (42). The hearts were stained with Rhod-2 AM (1.4 $\mu \mathrm{mol} / 1$, from Invitrogen) for $\mathrm{Ca}_{\mathrm{i}}$ mapping and $\mathrm{RH} 237$ (10 $\mu \mathrm{mol} / 1$, from Invitrogen) for $\mathrm{V}_{\mathrm{m}}$ mapping. Blebbistatin $(15-20 \mu \mathrm{mol} / 1$, from Tocris Bioscience) was used to inhibit contraction. The hearts were excited with a laser (Verdi G5, Coherent Inc.) at a wavelength $532 \mathrm{~nm}$. The fluorescence was recorded with $100 \times 100$ pixels for a spatial resolution of $0.35 \times 0.35 \mathrm{~mm}^{2}$ per pixel at $2 \mathrm{~ms} /$ frame sampling rate. Optical signals were processed with both spatial ( $3 \times 3$ pixels Gaussian filter) and temporal ( 3 frames moving average) filtering.

Mapping protocol. Four different protocols were used and are listed below. Among them, protocol I was performed to test if $I_{\text {KAS }}$ activation by CyPPA $(10 \mu \mathrm{mol} / 1)$ induces JWS and if $I_{\text {KAS }}$ blockade by apamin $(100$ $\mathrm{nmol} / \mathrm{l})$ is antiarrhythmic. In protocol II, we aimed to differentiate the effects of $I_{\mathrm{KAS}}$ carried by different SK channel isoforms. Apamin (100 nmol/1) blocked $I_{\text {KAS }}$ conducted by both SK2 and SK3, while Lei-Dab 7 at $15 \mathrm{nmol} / 1$ (a highly specific SK2 blocker; ref. 16) only specifically blocked $I_{\mathrm{KAS}}$ carried by SK2. In protocol III, isoproterenol $(100 \mathrm{nmol} / \mathrm{l})$ was applied to test if this clinical therapeutic approach for $\mathrm{BrS}$ is effective in the CyPPA-induced JWS model. Protocols I-III were performed at $38.3^{\circ} \mathrm{C}$, which is the normal body temperature of rabbits (43). In protocol IV, hypothermia $\left(32.0^{\circ} \mathrm{C}\right)$ was created to test the participation of $I_{\mathrm{KAS}}$ in acquired JWS. A previous study showed that $I_{\mathrm{KAS}}$ is upregulated in hypothermia (44).

When comparing APD among baseline and after each treatment, we paced the RV or LV at fixed PCL (S1S1) to avoid rate-dependent APD changes. The slowest pacing rate is limited by the competing sinus or escape rates of the Langendorff-perfused hearts. At baseline before CyPPA, the sinus cycle length was approximately 320-400 ms. It was not possible to consistently pace the ventricles with PCL longer than the sinus cycle length. Similarly, CyPPA impaired AV conduction and induced premature beats. 
Therefore, it was difficult to perform atrial pacing or ventricular pacing at PCL shorter than $250 \mathrm{~ms}$ to stably achieve $1: 1$ AV conduction or $1: 1$ ventricular capture. Therefore, we chose to compare APD at 3 PCL: $250 \mathrm{~ms}, 280 \mathrm{~ms}$, and $300 \mathrm{~ms}$.

To observe spontaneous arrhythmias, we left the ventricles unpaced except during APD measurements. In some cases, P2R occurred during APD measurement with S1S1 pacing (Figure 6 after isoproterenol and Supplemental Figure 2). All other arrhythmia episodes occurred spontaneous during sinus or escape rhythm without pacing. We did not use programmed stimulation to induce arrhythmias. In all protocols, the drugs were sequentially added and recirculated in the perfusate.

Protocol I: Baseline- CyPPA- apamin in 14 hearts (8 males). Epicardial mapping with baseline- CyPPAapamin in 12 hearts (6 males). Two additional males were simultaneously mapped the epicardium and the endocardium of the RV. For the epicardial mapping, optical signals were obtained from the anterior surface of the heart including both the RV and the LV. For simultaneous epicardial and endocardial mapping, we made the RV flap preparation as previously described (45). To expose the endocardium, the right ventricular posterior wall was cut open and the RV flap was then created by cutting the posterior end of the RV free wall from the base to the apex along the posterior descending artery. Major arterial leaks were ligated to ensure continuous perfusion of the RV free wall. After baseline measurement, CyPPA was added to the perfusate. APD measurement was performed 10 minutes after CyPPA administration. Optical mapping was also attempted to capture the onset of SVF or SVT. Any VF or VT occurrence was allowed to continue for at most 180 seconds before electrical defibrillation. We also performed optical mapping during the defibrillation. Apamin was subsequently added to the perfusate in the presence of CyPPA recirculation. Fifteen minutes after apamin administration, another set of data was collected.

Protocol II: Epicardial mapping of both the RV and the LV. Protocol IIA: baseline- LeiDab7- CyPPAapamin in 3 hearts ( 2 males). Protocol IIB: in a changed sequence of agents as baseline- apamin- CyPPA in 3 hearts (2 males).

Protocol III: Epicardial mapping of both the RV and the LV with baseline- CyPPA- isoproterenol in 6 hearts (3 males).

Protocol IV: Epicardial mapping of both the RV and the LV with baseline $\left(38.3^{\circ} \mathrm{C}\right)$ - hypothermia $\left(32.0^{\circ} \mathrm{C}\right)$ - apamin $\left(\right.$ at $\left.32.0^{\circ} \mathrm{C}\right)$ - rewarming $\left(38.3^{\circ} \mathrm{C}\right)$ in 6 hearts $(3$ males). After baseline optical measurement at $38.3^{\circ} \mathrm{C}$, the temperature of the perfusate was decreased to $32.0^{\circ} \mathrm{C}$. The heart surface temperature was also measured to confirm the creation of hypothermia. The hypothermia was maintained stably at $32.0^{\circ} \mathrm{C} \pm 0.5^{\circ} \mathrm{C}$ for 15 minutes before the optical measurement. Apamin was subsequently added into the perfusate and recirculated at $32.0^{\circ} \mathrm{C}$. Fifteen minutes after apamin, another set of data was collected. The heart was then rewarmed to $38.3^{\circ} \mathrm{C}$. We paced the ventricle at PCL $300 \mathrm{~ms}$ for APD comparisons, at 150 $\mathrm{ms}$ for alternans induction at baseline, and $200 \mathrm{~ms}$ for alternans induction during hypothermia.

TMP recording. The TMP was recorded at the RVOT and the LV during CyPPA $(10 \mu \mathrm{mol} / \mathrm{l})$ administration in 4 hearts ( 2 males). After immobilization of the Langendorff-perfused hearts by blebbistatin, standard glass capillary microelectrodes filled with $3 \mathrm{~mol} / 1 \mathrm{KCl}$ with a tip resistance of approximately $20 \mathrm{M} \Omega$ at a digitization rate of $10 \mathrm{kHz}$ was used to record TMP. The data were acquired with an Axoscope 10.2 (Axon Instruments Inc.). The onset of SVF after CyPPA was recorded simultaneously by TMP and pECG. Experiments were performed at $38.3^{\circ} \mathrm{C}$.

Ventricular cardiomyocyte isolation. In order to perform patch clamp and confocal calcium imaging studies, ventricular cardiomyocytes were isolated according to previously descried methods (24). Briefly, after the sacrifice of rabbits, the hearts were rapidly excised and Langendorff perfused with oxygenated Tyrode's solution containing (in mmol/1): $138 \mathrm{NaCl}, 5.33 \mathrm{KCl}, 0.33 \mathrm{NaH}_{2} \mathrm{PO}_{4}, 1.18 \mathrm{MgCl}_{2}, 10 \mathrm{HEPES}, 10$ taurine, and 10 glucose, $\mathrm{pH} 7.4(\mathrm{NaOH})$, at $37^{\circ} \mathrm{C}$. After rinsing off remaining blood, the heart was perfused for $7-12$ minutes with Tyrode's solution containing liberase $(15 \mu \mathrm{g} / \mathrm{ml}$, Roche). Ventricles were then removed and the epicardium/subepicardium layers were carefully separated and minced into small pieces followed by dispersion with a large-bore glass pipette. The dispersed tissue was filtered through $100-\mu \mathrm{m}$ nylon mesh followed by centrifuging at $500 \mathrm{rpm}$ for 2 minutes at room temperature. The supernatant was removed and the myocytes were resuspended in Tyrode's solution containing $0.2 \mathrm{mmol} / 1 \mathrm{CaCl}_{2}$ and $1 \% \mathrm{BSA}$. The centrifugation was repeated and the myocytes were resuspended in Tyrode's solution containing $0.5 \mathrm{mmol} / 1 \mathrm{CaCl}_{2}$ and $1 \% \mathrm{BSA}$ until use.

Patch clamp studies to determine the effects of CyPPA on $I_{N a}$. A whole-cell patch clamp technique was performed as described elsewhere (46). Briefly, the isolated ventricular cardiomyocytes were superfused with bath solution containing (in mmol/1) $137 \mathrm{NaCl}, 5.4 \mathrm{KCl}, 1.0 \mathrm{CaCl}_{2}, 0.5 \mathrm{MgCl}_{2}, 10$ glucose, and $10 \mathrm{HEPES}$, 
$\mathrm{pH}$ 7.4. Patch pipette resistances varied from 2.5 to $3.0 \mathrm{M} \Omega$ when they filled with an internal solution containing (mmol/1) $20 \mathrm{CsCl}, 110$ Cs-aspartate, $0.5 \mathrm{MgCl}_{2}, 5 \mathrm{MgATP}, 0.5$ EGTA, and $10 \mathrm{HEPES}$, pH 7.2 by $\mathrm{CsOH}$ adjustment. While $I_{\mathrm{Na}}$ recording, $\mathrm{K}^{+}$in pipette solution was replaced with equal molar amounts of $\mathrm{Cs}^{+}$. Voltage protocol and data acquisition were controlled by Axopatch 200B amplifier/Digidata 1440A acquisition system using pCLAMP 10.4 software (Molecular Devices). Whole-cell currents were elicited by $40-\mathrm{ms}$ pulses from $-90 \mathrm{mV}$ up to $+50 \mathrm{mV}$ in $10-\mathrm{mV}$ increments at the holding potential of $-90 \mathrm{mV}$. Paired-pulse protocols for $I_{\mathrm{Na}}$ activation, inactivation, and recovery from inactivation were also performed. After baseline measurement, CyPPA $(10 \mu \mathrm{mol} / 1)$ was added and another set of data was collected. In addition, we also performed dose-response experiments by adding CyPPA with different concentrations ranging from 0.1 to $30 \mu \mathrm{mol} / 1$. Voltage-dependent $I_{\mathrm{Na}}$ and dose-response curves were fitted by pCLAMP 10.2 (Molecular Devices) and custom-made software. $I_{\mathrm{Na}}$ was recorded at room temperature.

Confocal calcium imaging. Ventricular cardiomyocytes were centrifuged and resuspended in $\mathrm{Ca}^{2+}$ free Tyrode's solution. The $\mathrm{Ca}^{2+}$ indicator dye Fluo-4AM (Invitrogen) was loaded for 20 minutes followed by centrifugation and resuspended in Tyrode's solution with $1.8 \mathrm{mM} \mathrm{Ca}^{2+}$. The dye-loaded myocytes were then placed in a chamber on the stage of an inverted microscope (Leica TCS SP8 LSCM) fitted with a $\times 401.42$ NA oil immersion objective. Myocytes were stimulated via 2 platinum electrodes on either side of the bath at $0.5 \mathrm{~Hz}$. The Fluo-4AM was excited at $488 \mathrm{~nm}$ wavelength with an argon/ krypton laser. Emission wavelengths above $510 \mathrm{~nm}$ were detected by photomultiplier. Fluorescence intensity space-time recordings with electrical stimulation were acquired in the line-scan mode (1.69 $\mathrm{ms} /$ line) along the longitudinal axis of the myocytes. After baseline recording, CyPPA $(10 \mu \mathrm{mol} / 1)$ and apamin $(100 \mathrm{nmol} / \mathrm{l})$ were sequentially added. The normalized fluorescence signal $\left(\mathrm{F} / \mathrm{F}_{0}\right)$ of $\mathrm{AP}$ induced calcium transient was analyzed.

$R N A$ preparation and $q P C R$. The ventricular tissues were dissected from the epicardium of RVOT and LVB, cut into small pieces in RNAlater (Applied Biosystems/Ambion) and immersed at $4^{\circ} \mathrm{C}$ overnight, then stored at $-80^{\circ} \mathrm{C}$. Total RNA was extracted from the tissues using random primers with RNAqueous 4PCR kit (Applied Biosystems/Ambion). cDNA was synthesized from 100 ng total RNA for each sample using an iScript Select cDNA Synthesis Kit (Bio-Rad). qPCR was performed using iQ SYBR Green Supermix with iCycler (Bio-Rad). Specific primers for SK3 were sense 5'-TTGCCCAACTCCAGAGC-3' and antisense 5'-CAAGCAAGTGGTCATTGAGATTTA-3' (47). The general protocol for amplification was $95^{\circ} \mathrm{C}$ for 20 seconds, $58^{\circ} \mathrm{C}$ for 20 seconds, and $72^{\circ} \mathrm{C}$ for 20 seconds, for 40 cycles. PCR products were analyzed in agarose gels, and the amplicons were gel purified with QIAquick Gel Extraction Kit (QIAGEN). The sequentially diluted SK3 amplicons were used for standard curves. Data were collected by MyiQ Software (Bio-Rad), and analyzed using the threshold cycle $\left(C_{t}\right)$ relative quantification method. The glyceraldehyde 3-phosphate dehydrogenase (GAPDH) reference gene was used for normalizing the data. $2^{-\triangle \mathrm{Ct}}$ corresponded to the ratio of SK3 versus GAPDH. The primers for GAPDH were sense 5'-CCATGGAGAAGGCCGGGG-3' and antisense 5'-CGAAGTGGTCGTGGATGAC-3' (6).

In vivo surface ECG recording. In vivo surface ECG recording was performed in 1 male and 1 female rabbit. Anesthesia was induced by isoflurane (2\%-5\%) inhalation. Ketamine (5-10 mg/kg) and xylazine (3-5 mg/ $\mathrm{kg}$ ) were mixed and administered by i.v. injection to maintain sedation. After anesthesia, the rabbits were intubated and ventilated ( 45 breaths $/ \mathrm{min}, 15 \mathrm{ml} / \mathrm{kg}$ ). The rabbits were shaved and a 9-lead ECG (V1-V6, I, II, and III) was continuously recorded throughout the entire experiment by needle electrodes. Rectal temperature was continuously monitored. We first recorded the ECG at normothermia (around $38.3^{\circ} \mathrm{C}$ ). Hypothermia was then induced by a temperature-controlled water blanket. Alcohol was sprayed on the abdomen to speed up the cooling process. The cooling system was turned off when the rectal temperature reached $34.0^{\circ} \mathrm{C}$. The rectal temperature continued to fall by $1^{\circ} \mathrm{C}$ to $2^{\circ} \mathrm{C}$ afterwards. Hypothermia at $32.0^{\circ} \mathrm{C} \pm 0.5^{\circ} \mathrm{C}$ was maintained for 15 minutes followed by a single dose of apamin $(40 \mu \mathrm{g}$, i.v.). Fifteen minutes after apamin injection, the rabbits were rewarmed by water blanket till the body temperature gradually raised to baseline. At the completion of rewarming, the rabbit was euthanized with sodium pentobarbitone overdose $(160 \mathrm{mg} / \mathrm{kg}$, i.v.).

Statistics. Continuous variables are expressed as mean \pm SEM. Paired Student's $t$ tests were used to compare 2 variables (such as the number of phase singularities in the RV and the LV) from the same heart. Unpaired $t$ tests were used to compare variables between 2 groups (such as $\mathrm{J}$ point amplitude between females and males). One-way ANOVAs with Tukey's post hoc test were used to compare the means among 3 or more different variables (such as the numbers of SVF at baseline, after CyPPA, and after apamin). Two-way ANOVAs with Bonferroni's post hoc test were used to compare variables among different time 
points (such as $\mathrm{APD}_{25}$ at baseline, after CyPPA, and after apamin), which measured at different PCL. A 2-sided $P$ value of less than 0.05 was considered statistically significant.

Study approval. All animal studies were approved by the IACUC of Indiana University School of Medicine, and conformed to the Guide for the Care and Use of Laboratory Animals (NIH).

\section{Author contributions}

MC contributed to the hypothesis, designed and performed experiments, analyzed data, and wrote and revised the manuscript. DZX, AZW, SG, JW, and DY performed experiments and analyzed data. SFL, ZC, MRL, THE, ZQ, and JNW contributed to designing and interpreting experiments, and revised the manuscript. PSC contributed to the hypothesis, directed the work, designed experiments, and wrote the manuscript. All authors reviewed and approved the final manuscript.

\section{Acknowledgments}

This work was supported by NIH grants (P01 HL78931, R56 HL71140, R42DA043391, TR002208-01 and R01 HL139829); a Charles Fisch Cardiovascular Research Award endowed by Dr. Suzanne B. Knoebel of the Krannert Institute of Cardiology; a Medtronic-Zipes Endowment; and the Indiana University HealthIndiana University School of Medicine Strategic Research Initiative. We also thank Nicole Courtney, Jin Guo, Jian Tan, and Christopher Corr for their assistance.

Address correspondence to: Peng-Sheng Chen, 1800 N. Capitol Avenue, E475, Indianapolis, Indiana 46202, USA. Phone: 317.274.0909; Email: chenpp@iu.edu.

1. Antzelevitch C, et al. J-Wave syndromes expert consensus conference report: Emerging concepts and gaps in knowledge. Heart Rhythm. 2016;13(10):e295-e324.

2. Priori SG, et al. HRS/EHRA/APHRS expert consensus statement on the diagnosis and management of patients with inherited primary arrhythmia syndromes: document endorsed by HRS, EHRA, and APHRS in May 2013 and by ACCF, AHA, PACES, and AEPC in June 2013. Heart Rhythm. 2013;10(12):1932-1963.

3. Murray KT, Anno T, Bennett PB, Hondeghem LM. Voltage clamp of the cardiac sodium current at 37 degrees $\mathrm{C}$ in physiologic solutions. Biophys J. 1990;57(3):607-613.

4. Liu B, Arlock P, Wohlfart B, Johansson BW. Temperature effects on the Na and Ca currents in rat and hedgehog ventricular muscle. Cryobiology. 1991;28(1):96-104.

5. Park DS, et al. Genetically engineered SCN5A mutant pig hearts exhibit conduction defects and arrhythmias. J Clin Invest. 2015;125(1):403-412.

6. Chua SK, et al. Small-conductance calcium-activated potassium channel and recurrent ventricular fibrillation in failing rabbit ventricles. Circ Res. 2011;108(8):971-979.

7. Zhang XD, Lieu DK, Chiamvimonvat N. Small-conductance $\mathrm{Ca}^{2+}$-activated $\mathrm{K}^{+}$channels and cardiac arrhythmias. Heart Rhythm. 2015;12(8):1845-1851.

8. Chang PC, Chen PS. SK channels and ventricular arrhythmias in heart failure. Trends Cardiovasc Med. 2015;25(6):508-514.

9. Yu CC, et al. KCNN2 polymorphisms and cardiac tachyarrhythmias. Medicine (Baltimore). 2016;95(29):e4312.

10. Ellinor PT, et al. Common variants in KCNN3 are associated with lone atrial fibrillation. Nat Genet. 2010;42(3):240-244

11. Mahida S, et al. Overexpression of KCNN3 results in sudden cardiac death. Cardiovasc Res. 2014;101(2):326-334

12. Hougaard C, et al. Selective positive modulation of the SK 3 and SK2 subtypes of small conductance $\mathrm{Ca}^{2+}$-activated $\mathrm{K}^{+}$channels. Br J Pharmacol. 2007;151(5):655-665.

13. Yu CC, Ai T, Weiss JN, Chen PS. Apamin does not inhibit human cardiac $\mathrm{Na}^{+}$current, L-type $\mathrm{Ca}^{2+}$ current or other major $\mathrm{K}^{+}$ currents. PLoS ONE. 2014;9(5):e96691.

14. Kawazoe H, et al. Risk stratification of ventricular fibrillation in Brugada syndrome using noninvasive scoring methods. Heart Rhythm. 2016;13(10):1947-1954.

15. Nademanee K, et al. Fibrosis, connexin-43, and conduction abnormalities in the Brugada syndrome. J Am Coll Cardiol. 2015;66(18):1976-1986.

16. Shakkottai VG, et al. Design and characterization of a highly selective peptide inhibitor of the small conductance calciumactivated $\mathrm{K}^{+}$channel, SkCa2. J Biol Chem. 2001;276(46):43145-43151.

17. Kaneko Y, et al. Electrical storm in patients with brugada sy ndrome is associated with early repolarization. Circ Arrhythm Electrophysiol. 2014;7(6):1122-1128.

18. Brugada R, Campuzano O, Sarquella-Brugada G, Brugada P, Brugada J, Hong K. Brugada syndrome. In: Adam MP, et al, eds. GeneReviews. University of Washington, Seattle; 1993.

19. Cho LT, et al. An intracellular allosteric modulator binding pocket in SK2 ion channels is shared by multiple chemotypes. Structure. 2018;26(4):533-544.e3.

20. Tuteja D, et al. Cardiac small conductance $\mathrm{Ca}^{2+}$-activated $\mathrm{K}^{+}$channel subunits form heteromultimers via the coiled-coil domains in the C termini of the channels. Circ Res. 2010;107(7):851-859.

21. Hancock JM, Weatherall KL, Choisy SC, James AF, Hancox JC, Marrion NV. Selective activation of heteromeric SK channels contributes to action potential repolarization in mouse atrial myocytes. Heart Rhythm. 2015;12(5):1003-1015. 
22. Zhang Q, et al. Functional roles of a $\mathrm{Ca}^{2+}$-activated $\mathrm{K}^{+}$channel in atrioventricular nodes. Circ Res. 2008;102(4):465-471.

23. Zhang $\mathrm{XD}$, et al. Critical roles of a small conductance $\mathrm{Ca}^{2+}$-activated $\mathrm{K}^{+}$channel (SK3) in the repolarization process of atrial myocytes. Cardiovasc Res. 2014;101(2):317-325.

24. Chan YH, et al. Small-conductance calcium-activated potassium current is activated during hypokalemia and masks short-term cardiac memory induced by ventricular pacing. Circulation. 2015;132(15):1377-1386.

25. Hsieh YC, et al. Apamin-sensitive potassium current modulates action potential duration restitution and arrhythmogenesis of failing rabbit ventricles. Circ Arrhythm Electrophysiol. 2013;6(2):410-418.

26. Morita $\mathrm{H}$, et al. Repolarization heterogeneity in the right ventricular outflow tract: correlation with ventricular arrhythmias in Brugada patients and in an in vitro canine Brugada model. Heart Rhythm. 2008;5(5):725-733.

27. Koncz I, et al. Mechanisms underlying the development of the electrocardiographic and arrhythmic manifestations of early repolarization syndrome. J Mol Cell Cardiol. 2014;68:20-28.

28. Yu CC, et al. Small conductance calcium-activated potassium current is important in transmural repolarization of failing human ventricles. Circ Arrhythm Electrophysiol. 2015;8(3):667-676.

29. Lukas A, Antzelevitch C. Differences in the electrophysiological response of canine ventricular epicardium and endocardium to ischemia. Role of the transient outward current. Circulation. 1993;88(6):2903-2915.

30. Hopenfeld B. Mechanism for action potential alternans: the interplay between L-type calcium current and transient outward current. Heart Rhythm. 2006;3(3):345-352.

31. Maoz A, Krogh-Madsen T, Christini DJ. Instability in action potential morphology underlies phase 2 reentry: a mathematical modeling study. Heart Rhythm. 2009;6(6):813-822.

32. Kennedy M, Bers DM, Chiamvimonvat N, Sato D. Dynamical effects of calcium-sensitive potassium currents on voltage and calcium alternans. J Physiol (Lond). 2017;595(7):2285-2297.

33. Shutt RH, Howlett SE. Hypothermia increases the gain of excitation-contraction coupling in guinea pig ventricular myocytes. Am J Physiol, Cell Physiol. 2008;295(3):C692-C700.

34. Reher TA, et al. Small-conductance calcium-activated potassium current in normal rabbit cardiac Purkinje cells. $J$ Am Heart Assoc. 2017;6(6):e005471.

35. Liang P, et al. Patient-specific and genome-edited induced pluripotent stem cell-derived cardiomyocytes elucidate single-cell phenotype of Brugada syndrome. J Am Coll Cardiol. 2016;68(19):2086-2096.

36. Zhang M, Meng XY, Cui M, Pascal JM, Logothetis DE, Zhang JF. Selective phosphorylation modulates the PIP2 sensitivity of the CaM-SK channel complex. Nat Chem Biol. 2014;10(9):753-759.

37. Simó-Vicens R, et al. A new negative allosteric modulator, AP14145, for the study of small conductance calcium-activated potassium (KCa 2) channels. Br J Pharmacol. 2017;174(23):4396-4408.

38. Turker I, et al. Amiodarone inhibits apamin-sensitive potassium currents. PLoS ONE. 2013;8(7):e70450

39. Ko JS, et al. Ondansetron blocks wild-type and p.F503L variant small-conductance $\mathrm{Ca}^{2+}$-activated $\mathrm{K}^{+}$channels. Am JPhysiol Heart Circ Physiol. 2018;315(2):H375-H88.

40. Wang HW, Yang ZF, Zhang Y, Yang JM, Liu YM, Li CZ. Beta-receptor activation increases sodium current in guinea pig heart. Acta Pharmacol Sin. 2009;30(8):1115-1122.

41. Salama G, Bett GC. Sex differences in the mechanisms underlying long QT syndrome. Am J Physiol Heart Circ Physiol. 2014;307(5):H640-H648.

42. Chang PC, Hsieh YC, Hsueh CH, Weiss JN, Lin SF, Chen PS. Apamin induces early afterdepolarizations and torsades de pointes ventricular arrhythmia from failing rabbit ventricles exhibiting secondary rises in intracellular calcium. Heart Rhythm. 2013;10(10):1516-1524.

43. Chen PH, White CE. Comparison of rectal, microchip transponder, and infrared thermometry techniques for obtaining body temperature in the laboratory rabbit (Oryctolagus cuniculus). J Am Assoc Lab Anim Sci. 2006;45(1):57-63.

44. Hsieh YC, et al. Apamin-sensitive potassium current modulate action potential duration and ventricular arrhythmia during therapeutic hypothermia in isolated rabbit hearts. Heart Rhythm. 2017;14(5):S-239, C-PO03-13. https://www.heartrhythmjour nal.com/article/S1547-5271(17)30427-7/pdf.

45. Tang L, et al. Intracellular calcium dynamics at the core of endocardial stationary spiral waves in Langendorff-perfused rabbit hearts. Am J Physiol Heart Circ Physiol. 2008;295(1):H297-H304.

46. Wu AZ, Loh SH, Cheng TH, Lu HH, Lin CI. Antiarrhythmic effects of (-)-epicatechin-3-gallate, a novel sodium channel agonist in cultured neonatal rat ventricular myocytes. Biochem Pharmacol. 2013;85(1):69-80.

47. Ozgen N, et al. Early electrical remodeling in rabbit pulmonary vein results from trafficking of intracellular SK2 channels to membrane sites. Cardiovasc Res. 2007;75(4):758-769. 